JOURNAL OF INTEGRAL EQUATIONS

AND APPLICATIONS

Volume 14, Number 1, Spring 2002

\title{
A GENERALIZED COLLECTIVELY COMPACT OPERATOR THEORY WITH AN APPLICATION TO INTEGRAL EQUATIONS ON UNBOUNDED DOMAINS
}

\author{
SIMON N. CHANDLER-WILDE AND BO ZHANG
}

Dedicated to Prof. Dr. Rainer Kress on the occasion of his 60th birthday.

\begin{abstract}
In this paper a generalization of collectively compact operator theory in Banach spaces is developed. A feature of the new theory is that the operators involved are no longer required to be compact in the norm topology. Instead it is required that the image of a bounded set under the operator family is sequentially compact in a weaker topology. As an application, the theory developed is used to establish solvability results for a class of systems of second kind integral equations on unbounded domains, this class including in particular systems of Wiener-Hopf integral equations with $L^{1}$ convolutions kernels.
\end{abstract}

1. Introduction. Consider operator equations of the form

$$
\psi+K \psi=\phi
$$

and their approximation by

$$
\psi_{n}+K_{n} \psi_{n}=\phi_{n},
$$

where $K_{n}$ and $K$ are bounded linear operators on $Y$ and $Y$ is a real or complex Banach space. Let $B(Y)$ denote the Banach space of bounded linear operators on $Y$ and let us assume that $\left(K_{n}\right) \subset B(Y)$ is collectively compact, $K \in B(Y)$ is compact, $\phi_{n} \rightarrow \phi$, and $K_{n} \chi \rightarrow K \chi$ for all $\chi \in Y$. Then, from collectively compact operator theory $[\mathbf{2}$, Theorem 1.6], it follows that

$$
\begin{aligned}
& (I-K)^{-1} \in B(Y) \Longleftrightarrow \text { for some } N \text { and all } n \geq N, \\
& \left(I-K_{n}\right)^{-1} \in B(Y) \text { and is bounded uniformly in } n .
\end{aligned}
$$

Received by the editors on June 22, 2001, and in revised form on January 11, 2002.

Copyright (C)2002 Rocky Mountain Mathematics Consortium 
Since $K$ is compact, (1.3) is equivalent to the result

$$
\begin{aligned}
& (I-K) \text { is injective } \Longleftrightarrow \text { for some } N \text { and all } n \geq N, \\
& \left(I-K_{n}\right)^{-1} \in B(Y) \text { and is bounded uniformly in } n .
\end{aligned}
$$

Further, if the righthand side of (1.4) holds, it follows that $\psi_{n}=$ $\left(I-K_{n}\right)^{-1} \phi_{n} \rightarrow \psi=(I-K)^{-1} \phi$.

There are many applications where $K \in B(Y)$ and $\left(K_{n}\right) \subset B(Y)$ are not compact in the norm topology on $Y$ but do have sequential compactness properties in a weaker topology, see, e.g., $[\mathbf{6}, \mathbf{4}, \mathbf{8}, \mathbf{1 0}$, 12]. A typical example is the case where $Y=B C(\mathbf{R})$, the Banach space of functions bounded and continuous on $\mathbf{R}$, and $K$ is the integral operator on $Y$ defined by

$$
K \psi(s)=\int_{\mathbf{R}} k(s, t) \psi(t) d t, \quad s \in \mathbf{R},
$$

with $k: \mathbf{R}^{2} \rightarrow \mathbf{C}$ satisfying that $k(s, \cdot) \in L^{1}(\mathbf{R}), s \in \mathbf{R}$. If

$$
\sup _{s \in \mathbf{R}} \int_{\mathbf{R}}|k(s, t)| d t<\infty
$$

and, for all $s \in \mathbf{R}$,

$$
\int_{\mathbf{R}}\left|k(s, t)-k\left(s^{\prime}, t\right)\right| d t \longrightarrow 0
$$

as $s^{\prime} \rightarrow s$, then $K$ is a bounded operator on $Y$, see $[\mathbf{4}, \mathbf{8}, \mathbf{1 0}]$, but is not necessarily compact. For example, if $k(s, t)=\kappa(s-t)$, $s, t \in \mathbf{R}$, for some $\kappa \in L^{1}(\mathbf{R})$, then $K$ has the continuous spectrum $\{0\} \cup\{\hat{\kappa}(\xi): \xi \in \mathbf{R}\}$ where $\hat{\kappa}$ is the Fourier transform of $\kappa$ and so is not compact. However $K$ does have a sequential compactness property in a weaker topology, the strict topology of Buck [7], in that $K$ maps every bounded set into a bounded equicontinuous subset of $Y$. Thus the image of each bounded sequence has a subsequence which converges in the strict topology on $Y$, in which a sequence $\left(\chi_{n}\right)$ is convergent if it is bounded, i.e., $\sup _{n}\left\|\chi_{n}\right\|_{\infty}<\infty$, and if $\chi_{n}(s) \rightarrow \chi(s)$ uniformly on every finite interval $[\mathbf{1 0}$, Lemma 2.2], see also $[\mathbf{4}, \mathbf{8}]$.

In [8] (1.4) is generalized to the integral operator $K$ defined by (1.5) in the case when $k(s, t)=\kappa(s-t) z(t)$ with $\kappa \in L^{1}(\mathbf{R}), z \in L^{\infty}(\mathbf{R})$. 
Denoting the integral operator $K$ in this case by $K_{z}$, the key idea in [8] is not to consider a single operator $K_{z}$, which on its own has insufficient properties, but to consider a whole family of operators $S=\left\{K_{z}: z \in W\right\}$, where $W$ is chosen to be translation invariant and sequentially compact in the weak star topology on $L^{\infty}(\mathbf{R})$ so that $S$ is translation invariant and has a collective compactness property with respect to sequential compactness in the strict topology on $Y$. In particular, for the case $W=\{z \in X$ : ess. range $z \subset Q\}$, with $Q \subset \mathbf{C}$ compact and convex, the following replacement for (1.4) is obtained $[8]$ :

(1.8) $I-K_{z}$ injective for all $z \in W \Longrightarrow I-K_{z}$

surjective for all $z \in W$ and $\left(I-K_{z}\right)^{-1}$ uniformly bounded in $z$.

This result is generalized to the multi-dimensional case in $[\mathbf{1 0}$, Theorem $2.8]$, and see $[\mathbf{4}, \mathbf{1 2}, \mathbf{1 4}]$ for related results.

In this paper we develop in Sections 2-4 a generalized collectively compact theory. This generalized theory includes results of standard collectively compact operator theory [2], in particular (1.4), and the results of $[\mathbf{8}, \mathbf{1 0}, \mathbf{1 2}]$, cf. (1.8), as special cases. A main feature of the new theory is that the image of a bounded set under the collectively compact family is required to be sequentially compact not in the norm topology but in a weaker topology-the $\beta$ topology introduced in Section 2. The second main feature is that the theory requires only weak notions of operator convergence (weaker than strong convergence with respect to the norm topology). These weaker notions of operator convergence are discussed in Section 3 and in the Appendix.

To illustrate the general theory, we apply it in Section 5 to establish solvability results for a class of systems of second kind integral equations on unbounded domains. This section has been written so as to be selfcontained as far as possible, and the reader interested in applications to integral equations may like to turn here first.

The results of Section 5 are applied in $[\mathbf{1 1}, \mathbf{1 8}]$ to problems of time harmonic wave scattering by unbounded rough interfaces and inhomogeneous layers. In these two papers Theorems 5.1 and 5.7 are applied to reformulations of the respective boundary value problems as systems of coupled boundary and domain integral equations. Specifically, they are used to deduce existence of solution and well-posedness, for 
both the integral equation systems and their equivalent boundary value problems, from uniqueness results for the boundary value problems.

A further, related application of results and ideas from Sections 2-4 to obtain existence and well-posedness is made in Arens [5], in which the problem of time harmonic elastic wave scattering by unbounded rough surfaces is studied via reformulation as a system of boundary integral equations.

We restrict our attention in this paper to linear operator equations and their approximation. The strict topology on $B C[0, \infty)$ and collective compactness with respect to sequential compactness in the strict topology have also played a role in the analysis of classes of nonlinear Fredholm integral equations on the half-line. The reader is referred to $[\mathbf{3}, \mathbf{1 3}, \mathbf{1}]$ where both existence results and convergence results for numerical solution schemes are obtained. It may be that ideas from Sections 2-4 prove helpful in developing results, analogous to those in $[3, \mathbf{1 3}, \mathbf{1}]$, for nonlinear operator equations in a more abstract setting.

2. The $\beta$ topology. Let $X$ denote a real or complex Fréchet space, and let $\left\{|\cdot|_{n}: n \in \mathbf{N}\right\}$ be a countable separating family of semi-norms on $X$ which generates the Fréchet space topology. (For definitions of the topological and functional analytic terminologies used, see, e.g., $[\mathbf{1 6}, \mathbf{1 5}, \mathbf{1 9}]$. We define the subspace $Y$ of $X$ by

$$
Y:=\left\{\phi \in X:\|\phi\|_{\infty}:=\sup _{n \in \mathbf{N}}|\phi|_{n}<\infty\right\}
$$

and note that

(i) $\|\cdot\|_{\infty}$ is a norm on $Y$;

(ii) from Theorem 2.1 (ii) below it follows that, with $\|\cdot\|_{\infty}, Y$ is a Banach space;

(iii) if $X$ is not a Banach space, then $Y$ is a strict subspace of $X$.

Note further that $Y$ may consist only of the zero element, for example, if $X=\{a f: a \in \mathbf{R}\}$ with $f: \mathbf{R} \rightarrow \mathbf{R}$ defined by $f(t)=t, t \in \mathbf{R}$ and $|\cdot|{ }_{n}$ defined by $|\phi|_{n}=\sup _{|t| \leq n}|\phi(t)|$. In this case $X$ is a Banach space so the converse of (iii) does not hold. Some more substantial examples are the following: 
Example 2.1. $X=C(\mathbf{R})$ with $|\cdot|_{n}$ defined by $|\phi|_{n}=\sup _{|t| \leq n}|\phi(t)|$, so $Y=B C(\mathbf{R})$.

Example 2.2. $X=C(\mathbf{R})$ with, for some $p \in \mathbf{R},|\cdot|_{n}$ defined by $|\phi|_{n}=\sup _{|t| \leq n}|\phi(t)|(1+|t|)^{p}$, so that $Y=\left\{\phi \in C(\mathbf{R}): \phi(s)=O\left(|s|^{-p}\right)\right.$ as $|s| \rightarrow \infty\}$.

Example 2.3. $X=\{\phi \in C(0, \infty): \phi(s)=O(1)$ as $s \rightarrow \infty\}$ with $|\cdot|_{n}$ defined by $|\phi|_{n}=\sup _{t \geq 1 / n}|\phi(t)|$, so $Y=B C(0, \infty)$.

Example 2.4. $X=L_{\mathrm{loc}}^{2}(\mathbf{R})$ with $|\cdot|_{n}$ defined by $|\phi|_{n}=$ $\left\{\int_{-n}^{n}|\phi(t)|^{2} d t\right\}^{1 / 2}$, so $Y=L^{2}(\mathbf{R})$.

Examples 2.1 and 2.2 illustrate that the subspace $Y$ constructed depends not just on $X$ and its topology but also on the choice of the semi-norms $|\cdot|_{n}$ to generate the topology on $X$.

We now define a further topology on $Y$ as follows. Let $S_{0}$ denote the set of positive null sequences,

$$
S_{0}:=\left\{\left(a_{1}, a_{2}, \ldots\right): a_{n}>0 \text { for each } n \text { and } a_{n} \rightarrow 0 \text { as } n \rightarrow \infty\right\} .
$$

For $a \in S_{0}$ define the semi-norm $|\cdot|_{a}$ on $Y$ by

$$
|\phi|_{a}:=\sup _{n \in \mathbf{N}} a_{n}|\phi|_{n} .
$$

Then $\left\{|\cdot|_{a}: a \in S_{0}\right\}$ is a separating family of semi-norms on $Y$ which, with the topology generated, is a locally convex Haussdorf topological vector space (TVS). Let us call this topology on $Y$ the $\beta$ topology, the original topology on $X$ the $\tau$ topology, and the topology on $Y$ generated by $\|\cdot\|_{\infty}$ the norm topology. Given $\phi \in Y$ and a sequence $\left(\phi_{n}\right) \subset Y$, we will write $\phi_{n} \rightarrow \phi$ if $\left\|\phi_{n}-\phi\right\|_{\infty} \rightarrow 0$ as $n \rightarrow \infty, \phi_{n} \stackrel{\beta}{\rightarrow} \phi$ if $\left(\phi_{n}\right)$ converges to $\phi$ in the $\beta$ topology, and $\phi_{n} \stackrel{\tau}{\rightarrow} \phi$ if $\left(\phi_{n}\right)$ converges to $\phi$ in the $\tau$ topology. It is easy to see that, as topologies on $Y$, the norm topology is finer than the $\beta$ topology, which is finer than the $\tau$ topology: i.e., every $\tau$-open set in $Y$ is $\beta$-open, and every $\beta$-open set is open in the norm topology. Thus, for $\left(\phi_{n}\right) \subset Y, \phi \in Y$,

$$
\phi_{n} \longrightarrow \phi \Longrightarrow \phi_{n} \stackrel{\beta}{\longrightarrow} \phi \Longrightarrow \phi_{n} \stackrel{\tau}{\longrightarrow} \phi .
$$


Remark 2.1. Suppose that $E$ is a locally compact topological space which is also $\sigma$-compact, i.e., $E=\cup_{n=1}^{\infty} E_{n}$, where $E_{1}, E_{2}, \ldots$, are compact. Let $X=C(E)$, the space of real- or complex-valued continuous functions on $E$, and define $|\phi|_{n}:=\sup _{s \in E_{n}}|\phi(s)|$, for $n \in \mathbf{N}$, $\phi \in C(E)$. Then $Y=B C(E)$, the space of bounded continuous functions on $E$, and $\beta$ is the strict topology of Buck [7]. In particular, as an example of such a space $E$, we can take $E$ to be any closed or open subset of a finite-dimensional Euclidean space, with the usual metric topology.

The following theorem, cf., Buck [7, Theorem 1], explores properties of the $\beta$ topology.

Theorem 2.1. (i) In $Y$ the bounded sets in the $\beta$ topology and the norm topology are the same.

(ii) If a sequence $\left(\phi_{n}\right) \subset Y$ is bounded in the norm topology and converges in the $\tau$ topology to $\phi \in X$, then $\phi \in Y$ and $\|\phi\|_{\infty} \leq$ $\sup _{n}\left\|\phi_{n}\right\|_{\infty}$.

(iii) On every $\beta$-bounded subset of $Y$ the $\beta$ topology coincides with the $\tau$ topology.

(iv) A sequence $\left(\phi_{n}\right) \subset Y$ is convergent in the $\beta$ topology if and only if $\left(\phi_{n}\right)$ is convergent in the (weaker) $\tau$ topology and is bounded in the norm topology.

(v) $Y$ is sequentially complete in the $\beta$ topology.

(vi) Either the $\beta$ topology is identical to the norm topology or the $\beta$ topology is not metrizable.

(vii) If $S \subset Y$ is $\beta$-bounded, then its $\beta$-closure and $\beta$-sequential closure coincide.

(viii) Every $\beta$-bounded subset of $Y$ is $\beta$-closed if and only if it is $\beta$-sequentially closed.

(ix) Unless the norm topology coincides with the $\beta$-topology, $(Y, \beta)$ is not barreled and is not a Baire space, and $Y$ is of the first category in $(Y, \beta)$.

Proof. It is clear that any bounded set in the norm topology is bounded in the $\beta$ topology. Conversely, if $S \subset Y$ were bounded in 
the $\beta$ topology but were not bounded in the norm topology, then there would exist $\left(\phi_{j}\right) \subset S$ and an increasing sequence $\left(n_{j}\right) \subset \mathbf{N}$ such that $0<\left|\phi_{j}\right|_{n_{j}}=\lambda_{j} \rightarrow \infty$ as $j \rightarrow \infty$. Choose $a \in S_{0}$ so that $a_{n_{j}}=\lambda_{j}^{-1 / 2}$. Then $\left|\phi_{j}\right|_{a} \geq a_{n_{j}}\left|\phi_{j}\right|_{n_{j}}=\lambda_{j}^{1 / 2} \rightarrow \infty$ as $j \rightarrow \infty$, which contradicts the boundedness in the $\beta$ topology of $S$.

If $\left(\phi_{n}\right) \subset Y$ is bounded in the norm topology, $\phi \in X$, and $\phi_{n} \stackrel{\tau}{\rightarrow} \phi$, then, for $m \in \mathbf{N},|\phi|_{m}=\left|\phi_{n}\right|_{m}+\left|\phi_{n}-\phi\right|_{m} \leq \sup _{j \in \mathbf{N}}\left\|\phi_{j}\right\|_{\infty}+\mid \phi_{n}-$ $\left.\phi\right|_{m} \rightarrow \sup _{j \in \mathbf{N}}\left\|\phi_{j}\right\|_{\infty}$ as $n \rightarrow \infty$. Thus $\phi \in Y$ and (ii) follows.

To see that (iii) holds, suppose $S \subset Y$ is $\beta$-bounded. A subbase of the $\beta$ topology on $Y$ is $\left\{b_{a, \phi}: a \in S_{0}, \phi \in Y\right\}$, where $b_{a, \phi}:=\left\{\psi \in Y:|\phi-\psi|_{a}<1\right\}$. Thus a sub-base of the $\beta$ topology on $S$ is $\beta_{S}:=\left\{S \cap b_{a, \phi}: a \in S_{0}, \phi \in Y\right\}$. It is enough to show that every element of $\beta_{S}$ is open in the $\tau$ topology on $S$. Since $S$ is $\beta$-bounded it is bounded in the norm topology by (i), so that $B:=\sup _{\psi \in S}\|\psi\|_{\infty}<\infty$. Suppose $a \in S_{0}, \phi \in Y, \tilde{\phi} \in S \cap b_{a, \phi}$. Choose $N \in \mathbf{N}$ such that $a_{n}<1 /\left[B+\|\phi\|_{\infty}\right]$ for all $n>N$, and set $\varepsilon:=1-|\phi-\tilde{\phi}|_{a}$ and $U:=\left\{\psi \in Y:|\tilde{\phi}-\psi|_{m}<\varepsilon / a_{m}, m=1, \ldots, N\right\}$. Then $U$ is $\tau$-open and $\tilde{\phi} \in S \cap U \subset S \cap b_{a, \phi}$. Thus every point $\tilde{\phi} \in S \cap b_{a, \phi}$ is an interior point in the $\tau$ topology on $S$, so that $S \cap b_{a, \phi}$ is $\tau$-open.

To prove (iv), note that, since every Cauchy sequence in a TVS is bounded [16], if $\left(\phi_{n}\right) \subset Y$ is convergent in the $\beta$ topology then it is bounded in the $\beta$ topology (and so bounded in the norm topology by (i)) and also convergent in the weaker $\tau$ topology. Conversely, suppose that $\phi_{n} \stackrel{\tau}{\rightarrow} \phi$ and $B:=\sup _{n \in \mathbf{N}}\left\|\phi_{n}\right\|_{\infty}<\infty$. Let $S:=\left\{\phi \in Y:\|\phi\|_{\infty} \leq B\right\}$. By (ii), $\phi \in S$ so that $\left(\phi_{n}\right)$ is convergent to $\phi$ in the $\tau$ topology on $S$. By (iii) it follows that $\left(\phi_{n}\right)$ is convergent to $\phi$ in the $\beta$ topology on $S$. Thus $\phi_{m} \stackrel{\beta}{\rightarrow} \phi$.

Suppose the sequence $\left(\phi_{n}\right) \subset Y$ is $\beta$-Cauchy. Then $\left(\phi_{n}\right)$ is $\tau$-Cauchy and so $\tau$-convergent to some $\phi \in X$, since $X$ is a Fréchet space. Since $\left(\phi_{n}\right)$ is $\beta$-Cauchy, it is also $\beta$-bounded and so bounded in the norm topology by (i). Thus, by (ii), $\phi \in Y$ and, by (iv), $\phi_{n} \stackrel{\beta}{\rightarrow} \phi$. Thus $Y$ is sequentially complete in the $\beta$ topology.

The proof of (vi) is as follows. If the $\beta$ topology is metrizable, then the $\beta$ topology and the norm topology are two complete metrizable topologies on the same vector space, with the $\beta$ topology weaker than the norm topology. By a standard deduction from the open mapping 
theorem, cf. [16, Corollary 2.12], the $\beta$ topology and the norm topology are the same.

To see that (vii) holds, suppose that $S \subset Y$ is $\beta$-bounded and that $\phi$ is a closure point of $S$ in the $\beta$ topology. Then $\phi$ is a closure point in the coarser $\tau$ topology. Since the $\tau$ topology is metrizable, it follows that there exists $\left(\phi_{n}\right) \subset S$ such that $\phi_{n} \stackrel{\tau}{\rightarrow} \phi$. Since $S$ is $\beta$-bounded and so norm-bounded by (i), it follows from (iv) that $\phi_{n} \stackrel{\beta}{\rightarrow} \phi$. Thus the $\beta$-closure of $S$ coincides with the $\beta$-sequential closure.

If $S \subset Y$ is $\beta$-bounded and $\beta$-sequentially closed, then its $\beta$-sequential closure is $S$ and so, by (vii), its $\beta$-closure is $S$. Thus $S$ is $\beta$-closed and (viii) follows.

To see (ix), consider the set $B=\{\phi \in Y:\|\phi\| \leq 1\} \subset Y$. Clearly, this set is balanced, convex, and absorbing. By (ii) and (iv) it is $\beta$ sequentially closed and, by (i) and (viii), it is also closed in the $\beta$ topology. Thus $B$ is a barrel in $(Y, \beta)$. But, in the $\beta$ topology, $B$ is not a neighborhood of any $\psi \in B$ (and so is nowhere dense) for $B$ fails to contain any nonempty finite intersection of the members of the sub-base $\left\{b_{a, \phi}: a \in S_{0}, \phi \in Y\right\}$. Thus $(Y, \beta)$ is not barrelled and hence [15] is not a Baire space. It follows from this, or directly from the representation $Y=\cup_{n=1}^{\infty} n B$, that $Y$ is of the first category in itself.

口

Let $\mathcal{L}(Y)$ denote the vector space of linear operators on $Y$. Let $C(Y)$, $S(Y)$ and $B(Y)$ denote the subspaces of $\mathcal{L}(Y)$ consisting of those linear operators which are, respectively, continuous, sequentially continuous, and bounded with respect to (w.r.t.) the $\beta$ topology. (Thus, in particular, $K \in S(Y)$ if and only if $K \in \mathcal{L}(Y)$ and $\psi_{n} \stackrel{\beta}{\rightarrow} \psi \Rightarrow K \psi_{n} \stackrel{\beta}{\rightarrow}$ $K \psi$, while $K \in B(Y)$ if and only if $K \in \mathcal{L}(Y)$ and $K$ maps $\beta$-bounded sets to $\beta$-bounded sets.) From standard properties of topological vector spaces [16, Theorems A6 and 1.30],

$$
C(Y) \subset S(Y) \subset B(Y) \subset \mathcal{L}(Y)
$$

Further, since a linear operator on a normed space is bounded if and only if it is continuous and, by Theorem 2.1(i), the $\beta$-bounded and norm-bounded subsets of $Y$ are identical, we see that $B(Y)$ is also the set of linear operators on $Y$ which are bounded/continuous with respect 
to the norm topology. Thus, every $K \in B(Y)$ has a finite norm $\|K\|$, defined by

$$
\|K\|=\sup _{\phi \in Y} \frac{\|K \phi\|_{\infty}}{\|\phi\|_{\infty}} .
$$

Remark 2.2. Even if $K \in B(Y)$ is compact with respect to the norm topology on $Y$, it does not follow that $K \in S(Y)$. A counterexample can be constructed as follows. As in Example 2.1 take $X=C(\mathbf{R})$, $|\phi|_{n}=\sup _{|t| \leq n}|\phi(t)|, Y=B C(\mathbf{R})$. Let $B C^{+}(\mathbf{R}):=\{\psi \in Y:$ $\lim _{t \rightarrow+\infty} \psi(t)$ exists $\}$. Then $B C^{+}(\mathbf{R})$ is a subspace of $\left(Y,\|\cdot\|_{\infty}\right)$ and, by the Hahn-Banach theorem, a bounded linear functional $\lambda: B C(\mathbf{R}) \rightarrow$ C exists such that

$$
\lambda \psi=\psi(+\infty), \quad \psi \in B C^{+}(\mathbf{R}) .
$$

Define $K: Y \rightarrow Y$ by $K \psi=\lambda \psi$. Then $K$ is compact on $Y$ with respect to the norm topology as it is bounded and has finite dimensional range. But $K$ is not sequentially continuous in the $\beta$ topology. To see this, for $n \in \mathbf{N}$, let $\psi_{n} \in B C^{+}(\mathbf{R})$ be such that $\psi_{n}(t)=0$ for $t \leq n,=1$ for $t \geq n+1$, and $\left\|\psi_{n}\right\|_{\infty}=1$. Then $\psi_{n} \stackrel{\beta}{\rightarrow} 0$ but $K \psi_{n}=1$ for each $n$.

A set $B \subset Y$ will be said to be bounded if it is bounded in the $\beta$ topology. Note that, by Theorem 2.1 (i), it then follows that $B \subset Y$ is bounded if and only if it is bounded in the norm topology. $B \subset Y$ will be said to be relatively sequentially compact in the $\beta$ topology if each sequence in $B$ has a subsequence converging in the $\beta$ topology to an element of $Y$. Clearly, if $B \subset Y$ is relatively sequentially compact in the $\beta$ topology, then $B$ is bounded.

The aim of this paper is to generalize the ideas of collectively compact operator theory by considering linear operators $K: Y \rightarrow Y$ and families of such operators which satisfy that

$$
\begin{aligned}
& B \subset Y \text { bounded } \Longrightarrow K(B) \text { is relatively } \\
& \text { sequentially compact in the } \beta \text { topology. }
\end{aligned}
$$

For such a linear operator $K$ we say that $K$ is sequentially compact with respect to the $\beta$ topology. Let us say that $\mathcal{K} \subset \mathcal{L}(Y)$ is collectively sequentially compact with respect to the $\beta$ topology if

$B \subset Y$ bounded $\Longrightarrow \cup_{K \in \mathcal{K}} K(B)$ is relatively sequentially compact in the $\beta$ topology. 
Clearly, if $K$ is sequentially compact with respect to the $\beta$ topology then $K \in B(Y)$, so that if $\mathcal{K}$ is collectively sequentially compact with respect to the $\beta$ topology, then $\mathcal{K} \subset B(Y)$ (in fact, $\mathcal{K}$ is uniformly bounded). Further, in view of Theorem 2.1(i) and (2.2), if $K$ is compact with respect to the norm topology on $Y$, then $K$ is sequentially compact with respect to the $\beta$ topology. It thus follows from Remark 2.2 that $K$ can be sequentially compact w.r.t. the $\beta$ topology while not being sequentially continuous.

3. Notions of operator convergence. A component in the arguments to be developed is that one needs some notion of the convergence of a sequence of operators. For $\left(K_{n}\right) \subset \mathcal{L}(Y), K \in \mathcal{L}(Y)$, let us write $K_{n} \stackrel{\beta}{\rightarrow} K$ if

$$
\phi_{n} \stackrel{\beta}{\longrightarrow} \phi \Longrightarrow K_{N} \phi_{n} \stackrel{\beta}{\longrightarrow} K \phi .
$$

Call $\mathcal{K} \subset \mathcal{L}(Y) \beta$-sequentially compact if, for every sequence $\left(K_{n}\right) \subset \mathcal{K}$, a subsequence $\left(K_{n_{m}}\right)$ and $K \in \mathcal{K}$ exist such that $K_{n_{m}} \stackrel{\beta}{\rightarrow} K$.

Note that $K \stackrel{\beta}{\rightarrow} K$ holds if and only if $K \in S(Y)$. It follows that, if $\mathcal{K} \subset \mathcal{L}(Y)$ is $\beta$-sequentially compact, then $\mathcal{K} \subset S(Y)$. As Remark 2.2 makes clear, there may exist operators $K \in B(Y)$ which are compact with respect to the norm topology but are not sequentially continuous with respect to the $\beta$ topology, so that $K \stackrel{\beta}{\rightarrow} K$ fails to be true.

A more familiar and related notion of operator convergence is that of strong (or pointwise) convergence. For $\left(K_{n}\right) \subset \mathcal{L}(Y), K \in \mathcal{L}(Y)$, we will say that $\left(K_{n}\right)$ converges to $K$ in the strong operator topology on $(Y, \beta)$, and write $K_{N} \stackrel{S}{\rightarrow} K$, if

$$
K_{n} \phi \stackrel{\beta}{\rightarrow} K \phi, \quad \phi \in Y .
$$

The following lemmas explore the properties of and relationship between these and other notions of operator convergence.

Lemma 3.1. Suppose $\left(K_{n}\right) \subset \mathcal{L}(Y), K \in \mathcal{L}(Y)$, and $K_{n} \stackrel{\beta}{\rightarrow} K$. Then

(i) $\psi_{m} \stackrel{\beta}{\rightarrow} 0$ as $m \rightarrow \infty \Rightarrow K_{n} \psi_{m} \stackrel{\beta}{\rightarrow} 0$ as $m, n \rightarrow \infty$. 
(ii) $K \in S(Y)$.

(iii) For some $N \in \mathbf{N}, K_{n} \in B(Y)$ for $n \geq N$, and

$$
\|K\| \leq \sup _{n \geq N}\left\|K_{n}\right\|<\infty .
$$

Proof. Suppose that (i) is false. Then there exists $\left(\psi_{m}\right) \subset Y$ with $\psi_{m} \stackrel{\beta}{\rightarrow} 0,\left(m_{j}\right) \subset \mathbf{N},\left(n_{j}\right) \subset \mathbf{N}$, with $m_{j} \rightarrow \infty, n_{j} \rightarrow \infty$ as $j \rightarrow \infty$, such that $K_{n_{j}} \psi_{m_{j}} \stackrel{\beta}{\rightarrow} 0$. But $\psi_{m_{j}} \stackrel{\beta}{\rightarrow} 0$ and $K_{n_{j}} \stackrel{\beta}{\rightarrow} 0$, giving a contradiction.

Suppose $\psi_{n} \stackrel{\beta}{\rightarrow} 0$. For every $a \in S_{0},\left|K \psi_{n}\right|_{a} \leq\left|K_{m} \psi_{n}\right|_{a}+\mid K \psi_{n}-$ $\left.K_{m} \psi_{n}\right|_{a}$. Given $\varepsilon>0$, by (i) we can choose $N \in \mathbf{N}$ such that $\left|K_{m} \psi_{n}\right|_{a}<\varepsilon / 2$ for $m, n \geq N$. Then $m \geq N$ can be chosen, keeping $n \geq N$ fixed, to make $\left|K \psi_{n}-K_{m} \psi_{n}\right|_{a}<\varepsilon / 2$. Thus $\left|K \psi_{n}\right|_{a} \rightarrow 0$ and so $K \psi_{n} \stackrel{\beta}{\rightarrow} 0$. We have shown that $K \in S(Y)$.

If (iii) is false then there exists $\left(\psi_{j}\right) \subset Y$ with $\left\|\psi_{j}\right\|_{\infty}=1$ and a subsequence $\left(K_{n_{j}}\right)$ of $\left(K_{n}\right)$ such that $a_{j}:=\left\|K_{n_{j}} \psi_{j}\right\|_{\infty} \rightarrow \infty$ as $j \rightarrow \infty$. Let $\phi_{j}:=a_{j}^{-1 / 2} \psi_{j}$. Then $\phi_{j} \rightarrow 0$ so that $\phi_{j} \stackrel{\beta}{\rightarrow} 0$ but $\left\|K_{n_{j}} \phi_{j}\right\|_{\infty}=a_{j}^{1 / 2} \rightarrow \infty$ so that, by Theorem 2.1(iv), $K_{n_{j}} \phi_{j} \stackrel{\beta}{\rightarrow} 0$. This contradicts that $K_{n_{j}} \stackrel{\beta}{\rightarrow} K$.

Lemma 3.2. Suppose $\left(K_{n}\right) \subset B(Y), K \in S(Y)$ and $\left\|K_{n}-K\right\| \rightarrow 0$. Then $K_{n} \stackrel{\beta}{\rightarrow} K$.

Proof. If $\psi_{n} \stackrel{\beta}{\rightarrow} \psi$, then $\sup _{n}\left\|\psi_{n}\right\|_{\infty}<\infty$ and $K_{n} \psi_{n}-K \psi=$ $\left(K_{n}-K\right) \psi_{n}+K\left(\psi_{n}-\psi\right)$. It follows that $\left\|\left(K_{n}-K\right) \psi_{n}\right\|_{\infty} \leq \| K_{n}-$ $K\left\|\sup _{n}\right\| \psi_{n} \|_{\infty} \rightarrow 0$. Thus, and since $K \in S(Y), K_{n} \psi_{n} \stackrel{\beta}{\rightarrow} K \psi$.

Note that, although Lemma 3.1 shows that $K_{n} \stackrel{\beta}{\rightarrow} K$ implies both that $K \in S(Y)$ and that $K_{n} \in B(Y)$ for all sufficiently large $n$, it is not necessarily the case that $K_{n} \in S(Y)$ for any $n \in \mathbf{N}$, unless $S(Y)=B(Y)$. For suppose that $K \in S(Y)$ and $\tilde{K} \in B(Y) \backslash S(Y)$ and 
set $K_{n}=K+\tilde{K} / n$. Then $K_{n} \notin S(Y)$ but $\left\|K_{n}-K\right\| \rightarrow 0$ so that $K_{n} \stackrel{\beta}{\rightarrow} K$, by Lemma 3.2 .

Lemma 3.3. If $\left(K_{n}\right) \subset B(Y)$ and $K_{n} \stackrel{S}{\rightarrow} K$, then $K \in B(Y)$ and

$$
\|K\| \leq \sup _{n \in \mathbf{N}}\left\|K_{n}\right\|_{\infty}<\infty .
$$

Proof. Since $Y$ is a Banach space, with the norm topology, and

$$
K_{n} \stackrel{S}{\rightarrow} K \Longrightarrow K_{n} \psi \stackrel{\beta}{\rightarrow} K \psi, \psi \in Y \Longrightarrow \sup _{n \in \mathbf{N}}\left\|K_{n} \psi\right\|_{\infty}<\infty, \quad \psi \in Y,
$$

by Theorem 2.1 (iv), the lemma follows from the Banach-Steinhaus theorem.

Lemma 3.4. Suppose $\left(K_{n}\right) \subset \mathcal{L}(Y), K \in \mathcal{L}(Y)$ and set $\tilde{K}_{n}:=$ $K_{n}-K$. Then $K_{n} \stackrel{\beta}{\rightarrow} K$ if and only if $K_{n} \stackrel{S}{\rightarrow} K, K \in S(Y)$ and $\tilde{K}_{n} \tilde{\psi}_{n} \stackrel{\beta}{\rightarrow} 0$ for every $\left(\tilde{\psi}_{n}\right) \subset Y$ with $\tilde{\psi}_{n} \stackrel{\beta}{\rightarrow} 0$.

Proof. We have seen in Lemma 3.1 that $K_{n} \stackrel{\beta}{\rightarrow} K \Rightarrow K \in S(Y)$, and clearly $K_{n} \stackrel{\beta}{\rightarrow} K$ implies that $K_{n} \stackrel{S}{\rightarrow} K$. If $\left(\psi_{n}\right) \subset Y, \psi \in Y$ and $\psi_{n} \stackrel{\beta}{\rightarrow} \psi$, then $\tilde{\psi}_{n}:=\psi_{n}-\psi \stackrel{\beta}{\rightarrow} 0$ and

$$
K_{n} \psi_{n}-K \psi=\tilde{K}_{n} \tilde{\psi}_{n}+\left(K_{n} \psi-K \psi\right)+\left(K \psi_{n}-K \psi\right) .
$$

The remainder of the lemma follows from this representation.

Let us say that $\mathcal{K} \subset \mathcal{L}(Y)$ is $\beta$-sequentially equicontinuous if

$$
\left(K_{n}\right) \subset \mathcal{K}, \psi_{n} \stackrel{\beta}{\rightarrow} 0 \Longrightarrow K_{n} \psi_{n} \stackrel{\beta}{\rightarrow} 0 .
$$

Clearly if $\mathcal{K}$ is $\beta$-sequentially equicontinuous, then $\mathcal{K} \subset S(Y)$ (suppose that $K \in \mathcal{K}$ and consider the sequence $\left(K_{n}\right)$ given by $\left.K_{n}=K, n \in \mathbf{N}\right)$. 
The significance of this definition in this context is the following corollary of Lemma 3.4.

Lemma 3.5. Suppose $\left(K_{n}\right) \subset S(Y), K \in S(Y)$. Then $K_{n} \stackrel{\beta}{\rightarrow}$ $K$ if and only if $K_{n} \stackrel{S}{\rightarrow} K$ and $\left\{K_{n}: n \in \mathbf{N}\right\}$ is $\beta$-sequentially equicontinuous.

Proof. It is shown in Lemma A.4 in the appendix that $\left\{K_{n}: n \in\right.$ $\mathbf{N}\} \subset S(Y)$ is $\beta$-sequentially equicontinuous if and only if $\psi_{n} \stackrel{\beta}{\rightarrow} 0 \Rightarrow$ $K_{n} \psi_{n} \stackrel{\beta}{\rightarrow} 0$. Thus the lemma follows from Lemma 3.4.

Let us say that $\mathcal{K} \subset \mathcal{L}(Y)$ is sequentially compact in the strong operator topology on $(Y, \beta)$, if, for every sequence $\left(K_{n}\right) \subset \mathcal{K}$, there exists $K \in \mathcal{K}$ and a subsequence $\left(K_{n_{m}}\right)$ such that $K_{n_{m}} \stackrel{S}{\rightarrow} K$. Then Lemma 3.5 and other observations made above imply the following corollary.

Corollary 3.6. Suppose $\mathcal{K} \subset \mathcal{L}(Y)$. Then $K$ is $\beta$-sequentially compact if and only if $\mathcal{K} \subset S(Y)$ and $\mathcal{K}$ is $\beta$-sequentially equicontinuous and sequentially compact in the strong operator topology on $(Y, \beta)$.

In the case that the $\beta$ and norm topologies coincide, in which case $C(Y)=S(Y)=B(Y)$, it follows from Lemma 3.3, in other words from the uniform boundedness theorem in Banach spaces, that if $K_{n} \stackrel{S}{\rightarrow} K$ and $\left(K_{n}\right) \subset B(Y)$, then $\left\{K_{n}: n \in \mathbf{N}\right\}$ is $\beta$-sequentially equicontinuous. In the case when these topologies do not coincide, in which case, by Theorem 2.1(vi), $(Y, \beta)$ is not metrizable, other versions of the Banach-Steinhaus theorem would apply $[\mathbf{1 6}, \mathbf{1 5}]$, if $(Y, \beta)$ were a Baire space or, more generally, a barrelled TVS, to give that $\left\{K_{n}: n \in \mathbf{N}\right\}$ is $\beta$-sequentially equicontinuous if $K_{n} \stackrel{S}{\rightarrow} K$ and $\left(K_{n}\right) \subset C(Y)$. But, by Theorem 2.1(ix), $(Y, \beta)$ is not barrelled unless the norm and $\beta$ topologies coincide. And in fact the following example makes it clear that a version of the Banach-Steinhaus theorem, enabling equicontinuity to be deduced from continuity and pointwise boundedness, does not always hold for $(Y, \beta)$ if the $\beta$ and norm 
topologies do not coincide.

Example 3.1. Let $Y$ be defined as in Example 2.1. For $n \in \mathbf{N}$ define $K_{n} \in \mathcal{L}(Y)$ by $K_{n} \psi=\psi(n), \psi \in Y$. It is easy to see that $\left(K_{n}\right) \subset C(Y) \subset B(Y)$. Also, $\sup _{n \in \mathbf{N}}\left\|K_{n} \psi\right\|_{\infty}<\infty, \psi \in Y$. It follows from the Banach-Steinhaus theorem applied to $Y$ with the norm topology that $\left(K_{n}\right)$ is uniformly bounded. But $\left(K_{n}\right)$ is not $\beta$ sequentially equicontinuous as, defining $\psi_{n}(s)=1+\tanh (s-n), s \in \mathbf{R}$, $n \in \mathbf{N}$, clearly $\left(\psi_{n}\right) \subset Y, \psi_{n} \stackrel{\beta}{\rightarrow} 0$, but $K_{n} \psi_{n}(0)=1$, so $K_{n} \psi_{n} \stackrel{\beta}{\nrightarrow} 0$.

In the case that $Y$ satisfies an additional assumption, we show below that it does hold that a sequence $\left(K_{n}\right) \subset S(Y)$ that is convergent in the strong operator topology on $(Y, \beta)$ is $\beta$-sequentially equicontinuous. For $m \in \mathbf{N}$, define the semi-norm $|\cdot|_{m}^{\prime}$ on $X$ by

$$
|\phi|_{m}^{\prime}:=\max _{1 \leq n \leq m}|\phi|_{n}
$$

and let $Y_{m}$ denote the linear subspace of $Y, Y_{m}:=\left\{\phi \in Y:|\phi|_{m}^{\prime}=0\right\}$. The additional assumption is the following one:

Assumption A. For every $m \in \mathbf{N}$ there exists $n>m$ and $P: Y \rightarrow$ $Y_{m}$ such that

$$
\|\psi-P \psi+\chi\|_{\infty} \leq \max \left(|\psi|_{n}^{\prime},\|\chi\|_{\infty}\right), \quad \psi \in Y, \chi \in Y_{n}
$$

That Assumption A is often satisfied in applications is illustrated by the following examples.

Example 3.2. Suppose that $Y$ and $|\cdot|_{n}$ are defined as in Example 2.1. Let $\phi \in C(\mathbf{R})$ be such that $0 \leq \phi(s) \leq 1, s \in \mathbf{R}, \phi(s)=1,|s| \leq m$, $\phi(s)=0,|s|>m+1$. Define $P: Y \rightarrow Y_{m}$ by

$$
P \psi=(1-\phi) \psi
$$

Then $\psi-P \psi=\phi \psi$ and (3.2) holds with $n=m+1$. 
Example 3.3. Generalizing Example 3.2, consider the case discussed in Remark 2.1, in which $X=C(E), Y=B C(E)$, with $E$ locally compact and $\sigma$-compact. Since a topological space is locally compact if and only if every compact set is contained in an open set with compact closure, it is easy to see that $E$ must also be regularly $\sigma$-compact, i.e., $E=\cup_{n=1}^{\infty} E_{n}$ with each $E_{n}$ compact and $E_{n} \subset O_{n} \subset E_{n+1}$ for some open set $O_{n}$. Then applying Urysohn's lemma [17], there exists $\phi \in C(E)$ with $0 \leq \phi(s) \leq 1, \phi(s)=1, s \in E_{m}, \phi(s)=0, s \in E \backslash E_{m+1}$. With the semi-norms $|\cdot|_{m}$ defined as in Remark 2.1 and defining $P$ again by (3.3), it follows that (3.2) holds with $n=m+1$.

The above construction shows that Assumption A is satisfied in Example 2.1 and in the case discussed in Remark 2.1. The same or a very similar argument applies to show that Assumption A holds in all the other examples we have considered so far.

Clearly, since $0 \in Y_{n}$, we see that

$$
\mathrm{A} \Longrightarrow \mathrm{A}^{\prime}
$$

where $\mathrm{A}^{\prime}$ is the following, closely related assumption:

Assumption $\mathrm{A}^{\prime}$. For every $m \in \mathbf{N}$ there exists $n>m$ and $P: Y \rightarrow Y_{m}$ such that

$$
\|\psi-P \psi\|_{\infty} \leq|\psi|_{n}^{\prime}, \quad \psi \in Y
$$

In the case that the $\beta$ and norm topologies coincide, it is easy to see that $Y_{n}=\{0\}$ for all sufficiently large $n$. In this case $\mathrm{A}$ is equivalent to $\mathrm{A}^{\prime}$ and $\mathrm{A}^{\prime}$ reduces to the assumption that, for some $n \in \mathbf{N}$,

$$
\|\psi\|_{\infty} \leq|\psi|_{n}^{\prime}, \quad \psi \in Y
$$

If the $\beta$ and norm topologies do not coincide, then there exists $\left(\psi_{m}\right) \subset$ $Y$ with $\left\|\psi_{m}\right\|_{\infty}=1$ and $\psi_{m} \stackrel{\beta}{\rightarrow} 0$. For such a sequence it holds that for every $n \in \mathbf{N}, 1=\left\|\psi_{m}\right\|_{\infty}>\left|\psi_{m}\right|_{n}^{\prime}$ for all sufficiently large $m$, contradicting (3.4). Thus, in the case that the $\beta$ and norm topologies do not coincide,

$$
\mathrm{A}^{\prime} \Longrightarrow Y_{m} \neq\{0\}, \quad m \in \mathbf{N}
$$


A further significance of Assumption $\mathrm{A}^{\prime}$ is the following result, the proof of which is postponed to the appendix.

Theorem 3.7. If Assumption $\mathrm{A}^{\prime}$ holds, then $C(Y)=S(Y)$.

Note that, although $C(Y)=S(Y)$ when Assumption $\mathrm{A}^{\prime}$ holds, Remark 2.2 and Example 3.2 together show that $\mathrm{A}^{\prime}$ does not imply that $S(Y)=B(Y)$.

The main importance of Assumption A for our purposes is the following result. The rather technical proof is postponed to the appendix.

Theorem 3.8. Suppose that Assumption A holds and that $\left(K_{n}\right) \subset$ $S(Y), K \in S(Y)$ and $K_{n} \stackrel{S}{\rightarrow} K$. Then $\left\{K_{n}: n \in \mathbf{N}\right\}$ is $\beta$-sequentially equicontinuous.

As a corollary of Theorem 3.8 and Lemma 3.5 we have the following result which shows that the convergence $K_{n} \stackrel{\beta}{\rightarrow} K$ is implied by strong convergence with respect to the $\beta$ topology if also $K_{n}$ and $K$ are sequentially continuous.

Corollary 3.9. Suppose that Assumption A holds and that $\left(K_{n}\right) \subset$ $S(Y), K \in S(Y)$. Then

$$
K_{n} \stackrel{\beta}{\rightarrow} K \Longleftrightarrow K_{n} \stackrel{S}{\rightarrow} K
$$

4. A generalized collectively compact operator theory. With the notions of collective compactness and operator convergence introduced in Sections 2 and 3 we can already state generalizations of some of the results in $[\mathbf{8}, \mathbf{1 0}, \mathbf{1 2}]$. But to generalize many of the results we need something playing the role that the translation operators play in $[8,10,12]$.

Let iso $(Y)$ denote the set of isometric isomorphisms on $Y$. Call $S \subset i s o(Y)$ sufficient if, for some $J \in \mathbf{N}$ it holds that, for every $\psi \in Y$, there exists $T \in S$ such that $\|\psi\|_{\infty} \leq 2|T \psi|_{J}$. The following examples illustrate this definition: 
Example 4.1. Define $X$ and $|\cdot|_{n}$ as in Example 2.1 so that $Y=B C(\mathbf{R})$. Let $S:=\left\{T_{n}: n \in \mathbf{Z}\right\}$ where, for $b \in \mathbf{R}, T_{b}: Y \rightarrow Y$ is the translation operator defined by

$$
T_{b} \psi(s)=\psi(s-b), \quad s \in \mathbf{R} .
$$

Then $S$ is sufficient (it satisfies the definition with $J=1$ ).

Example 4.2. Define $X$ and $|\cdot|_{n}$ as in Example 2.3 so that $Y=B C(0, \infty)$. Let $S:=\left\{M_{a}: a>0\right\}$ where, for $a>0, M_{a}: Y \rightarrow Y$ is defined by $M_{a} \psi(s)=\psi(a s), s>0$. Then $S$ is sufficient.

Example 4.3. Suppose $X=Y$ and $|\cdot|_{n}=\|\cdot\|_{\infty}, n \in \mathbf{N}$. Let $S:=\{I\}$ ( $I$ the identity operator). Then $S$ is sufficient.

The first result we give in this section is a generalization of a collectively compact operator approximation result of Anselone in [2, Theorem 1.6].

Remark 3.1. Note that, in Theorem 4.1 and subsequent theorems in this section, we require the first notion of convergence of a sequence of operators that we introduced in Section 2, that $K_{n} \stackrel{\beta}{\rightarrow} K$ if and only if (3.1) holds. We remind the reader that we have related this convergence to the more standard notion of convergence in the strong operator topology on $(Y, \beta)$ in the lemmas and corollaries 2.3-2.10. In particular, if Assumption A holds and $\left(K_{n}\right) \subset S(Y), K \in S(Y)$, then Corollary 2.10 shows that $K_{n} \stackrel{\beta}{\rightarrow} K$ if and only if $K_{n} \stackrel{S}{\rightarrow} K$. This may be helpful as it may be much easier to establish $K_{n} \stackrel{S}{\rightarrow} K$ than directly that $K_{n} \stackrel{\beta}{\rightarrow} K$.

In the following theorem we use the notation $\mathcal{K}^{\times}$to denote the set of subsequences of $\mathcal{K}_{1} \times \mathcal{K}_{2} \times \ldots$ (As usual, $\mathcal{K}_{1} \times \mathcal{K}_{2} \times \ldots$ denotes the set of sequences $\left(K_{n}\right)$ such that $\left.K_{1} \in \mathcal{K}_{1}, K_{2} \in \mathcal{K}_{2}, \ldots\right)$. We note that every subsequence of every sequence in $\mathcal{K}^{\times}$is again in $\mathcal{K}^{\times}$.

Theorem 4.1. Suppose that $\mathcal{K}, \mathcal{K}_{n} \subset \mathcal{L}(Y)$ for $n \in \mathbf{N}$ and that:

(i) $\cup_{n=1}^{\infty} \mathcal{K}_{n}$ is collectively sequentially compact with respect to the $\beta$ topology; 
(ii) for every sequence $\left(K_{n}\right) \in \mathcal{K}^{\times}$there exists a subsequence $\left(K_{n_{m}}\right)$ and $K \in \mathcal{K}$ such that $K_{n_{m}} \stackrel{\beta}{\rightarrow} K$;

(iii) $S \subset$ iso $(Y)$ is sufficient and, for all $n \in \mathbf{N}, K \in \mathcal{K}_{n}$, and $T \in S$, it holds that $T K T^{-1} \in \mathcal{K}_{n}$;

(iv) $I-K$ is injective for all $K \in \mathcal{K}$.

Then for some $N \in \mathbf{N}, I-K$ is injective for all $K \in \mathcal{K}_{n}, n \geq N$, so that $(I-K)^{-1}$ exists as an operator on the range space $(I-K)(Y)$ and

$$
\sup _{\substack{K \in \mathcal{K}_{n} \\ n \geq N}}\left\|(I-K)^{-1}\right\|<\infty .
$$

Proof. Suppose that the conclusion is false. Then for every $n \in \mathbf{N}$ and $\varepsilon>0$, there exists $n \geq N, \tilde{K} \in \mathcal{K}_{n}$ and $\phi \in Y$ with $\|\phi\|_{\infty}=1$ such that $\|(I-\tilde{K}) \phi\|_{\infty}<\varepsilon$. Thus there exist sequences $\left(\tilde{K}_{n}\right) \in \mathcal{K}^{\times}$ and $\left(\phi_{n}\right) \subset Y$ with $\left\|\phi_{n}\right\|_{\infty}=1$ such that

$$
\left(I-\tilde{K}_{n}\right) \phi_{n} \longrightarrow 0
$$

as $n \rightarrow \infty$. Since, by (iii), $S$ is sufficient, for some $J \geq 1$ and all $n \in \mathbf{N}$ there exists $T_{n} \in S$ such that $\left|T_{n} \phi_{n}\right|_{J} \geq(1 / 2)\left\|\phi_{n}\right\|_{\infty}=1 / 2$. Now let $\psi_{n}=T_{n} \phi_{n}$ and $K_{n}=T_{n} \tilde{K}_{n} T_{n}^{-1}$. For all $n \in \mathbf{N}, \tilde{K}_{n} \in \mathcal{K}_{m}$ for some $m \in \mathbf{N}$ and also $K_{n} \in \mathcal{K}_{m}$ by (iii). Thus $\left(K_{n}\right) \in \mathcal{K}^{\times}$, and, by (4.3) and the fact that $T_{n} \in$ iso $(Y)$, we have

$$
\left\|\psi_{n}-K_{n} \psi_{n}\right\|_{\infty}=\left\|T_{n}\left(I-\tilde{K}_{n}\right) \phi_{n}\right\|_{\infty}=\left\|\left(I-\tilde{K}_{n}\right) \phi_{n}\right\|_{\infty} \longrightarrow 0
$$

as $n \rightarrow \infty$. By (ii) we can find a subsequence $\left(K_{n_{m}}\right)$ and $K \in \mathcal{K}$ such that $K_{n_{m}} \stackrel{\beta}{\rightarrow} K$. By (i) we can find subsequences of $\left(\psi_{n_{m}}\right)$ and $\left(K_{n_{m}}\right)$ (denoted again by $\left(\psi_{n_{m}}\right)$ and $\left(K_{n_{m}}\right)$ ) and $\psi \in Y$ such that $K_{n_{m}} \psi_{n_{m}} \stackrel{\beta}{\rightarrow} \psi$. It follows from (4.4) and (2.2) that $\psi_{n_{m}} \stackrel{\beta}{\rightarrow} \psi$ and, since $K_{n_{m}} \stackrel{\beta}{\rightarrow} K$, it holds that $K_{n_{m}} \psi_{n_{m}} \stackrel{\beta}{\rightarrow} K \psi$. Thus $\psi=K \psi$. Now $\left|\psi_{n}\right|_{J} \geq 1 / 2$ and, by (2.2), $\psi_{n} \stackrel{\tau}{\rightarrow} \psi$ so that $\left|\psi_{n}-\psi\right|_{J} \rightarrow 0$. Thus $|\psi|_{J} \geq 1 / 2$ and, since $I-K$ is injective by (iv), we have a contradiction.

$\square$

The next two results, cf. $[\mathbf{4}, \mathbf{1 0}, \mathbf{1 2}]$, will be used to establish conditions, additional to those in Theorem 4.2, which will also ensure 
that $I-K$ is surjective so that $(I-K)^{-1} \in B(Y)$ for each $K \in \mathcal{K}$. Theorem 4.3 also establishes the convergence $\left(I-K_{n}\right)^{-1} \stackrel{\beta}{\rightarrow}(I-K)^{-1}$ in the case that $K_{n} \stackrel{\beta}{\rightarrow} K$ and the operators $\left(I-K_{n}\right)^{-1}$ are uniformly bounded.

Lemma 4.2. Suppose $\phi, \phi_{n} \in Y, K, K_{n} \in \mathcal{L}(Y)$ for $n \in \mathbf{N}$, $\left\{K_{n}: n \in \mathbf{N}\right\}$ is collectively sequentially compact with respect to the $\beta$ topology, $K_{n} \stackrel{\beta}{\rightarrow} K, \phi_{n} \stackrel{\beta}{\rightarrow} \phi, I-K$ is injective, and $\left(\psi_{n}\right) \subset Y$ is bounded and satisfies $\psi_{n}=\phi_{n}+K_{n} \psi_{n}$ for $n \in \mathbf{N}$. Then $\phi \in(I-K)(Y)$ so that $\psi:=(I-K)^{-1} \phi$ exists, and $\psi_{n} \stackrel{\beta}{\rightarrow} \psi$.

Proof. Since $\left(K_{n}\right)$ is collectively sequentially compact and $\left(\psi_{n}\right)$ is bounded, there exist subsequences $\left(\phi_{n_{m}}\right)$ and $\left(K_{n_{m}} \psi_{n_{m}}\right)$ such that $\phi_{n_{m}}+K_{n_{m}} \psi_{n_{m}} \stackrel{\beta}{\rightarrow} \psi \in Y$. Thus $\psi_{n_{m}} \stackrel{\beta}{\rightarrow} \psi$ and, since $K_{n} \stackrel{\beta}{\rightarrow}$ $K, K_{n_{m}} \psi_{n_{m}} \stackrel{\beta}{\rightarrow} K \psi$. Thus $\psi=\phi+K \psi$.

The above argument shows that $\phi \in(I-K)(Y)$ and that $\left(\psi_{n}\right)$ has a subsequence converging to $\phi=(I-K)^{-1} \phi$. The same argument shows that every subsequence of $\left(\psi_{n}\right)$ has a subsequence converging to $\psi$, so that $\psi_{n} \stackrel{\beta}{\rightarrow} \psi$.

Theorem 4.3. Let $K, K_{n} \in \mathcal{L}(Y)$ for $n \in \mathbf{N}$. Suppose $\left\{K_{n}: n \in\right.$ $\mathbf{N}\}$ is collectively sequentially compact with respect to the $\beta$ topology, $K_{n} \stackrel{\beta}{\rightarrow} K, I-K$ is injective, and $\left(I-K_{n}\right)^{-1} \in B(Y)$ for $n \in \mathbf{N}$, with $C:=\sup _{n \in \mathbf{N}}\left\|\left(I-K_{n}\right)^{-1}\right\|<\infty$. Then $(I-K)^{-1} \in B(Y)$, $\left\|(I-K)^{-1}\right\| \leq C$ and $\left(I-K_{n}\right)^{-1} \stackrel{\beta}{\rightarrow}(I-K)^{-1}$.

Proof. Since $I-K$ is injective, $(I-K)^{-1}$ exists as an operator on $(I-K)(Y)$.

Define the sequence $\left(\psi_{n}\right) \subset Y$ by $\psi_{n}:=\left(I-K_{n}\right)^{-1} \phi_{n}$. Then $\left(\psi_{n}\right)$ is bounded and $\psi_{n}=\phi_{n}+K_{n} \psi_{n}$, so that, by Lemma 4.2, $\phi \in(I-K)(Y)$ and $\psi_{n} \stackrel{\beta}{\rightarrow} \psi:=\left(I-K_{k}\right)^{-1} \phi$. Thus $I-K$ is surjective and $\left(I-K_{n}\right)^{-1} \phi_{n} \stackrel{\beta}{\rightarrow}(I-K)^{-1} \phi$. From this, (2.2) and Theorem 2.1 (ii), it follows, by taking $\phi_{n}=\phi$ for all $n$, that $\left\|(I-K)^{-1}\right\| \leq C$. 
The next result, cf. [4], [10, Theorem 2.7] and [8, Theorem 4.4] establishes conditions, additional to those in Theorem 4.2, which will ensure that $I-K$ is also surjective so that $(I-K)^{-1}: Y \rightarrow Y$ exists and is bounded for all $K \in \mathcal{K}$.

Theorem 4.4. Suppose that the conditions of Theorem 4.1 are satisfied and that, in addition, for every $K \in \mathcal{K}$, there exists $\left(K_{n}\right) \in \mathcal{K}^{\times}$ such that $K_{n} \stackrel{\beta}{\rightarrow} K$ and

$$
I-K_{n} \text { injective } \Longrightarrow I-K_{n} \text { surjective }
$$

for $n=1,2, \ldots$. Then for all $K \in \mathcal{K}, I-K$ is surjective so that $(I-K)^{-1}: Y \rightarrow Y$ exists and is bounded and, for some $n \in \mathbf{N}$,

$$
\sup _{K \in \mathcal{K}}\left\|(I-K)^{-1}\right\| \leq \sup _{\substack{K \in \mathcal{K}_{n} \\ n \geq N}}\left\|(I-K)^{-1}\right\|<\infty .
$$

Proof. Suppose $K \in \mathcal{K}$ and $\left(K_{n}\right) \in \mathcal{K}^{\times}$with $K_{n} \stackrel{\beta}{\rightarrow} K$ and (4.5) holding. By Theorem 4.1 there exists $N \in \mathbf{N}$ such that $I-\tilde{K}$ is injective for all $\tilde{K} \in \mathcal{K}_{n}$ and $n \geq N$ and such that

$$
C:=\sup _{\substack{\tilde{K} \in K_{n} \\ n \geq N}}\left\|(I-\tilde{K})^{-1}\right\|<\infty .
$$

Thus $\left(I-K_{n}\right)^{-1} \in B(Y)$ for $n \geq N$, with $\sup _{n \geq N}\left\|\left(I-K_{n}\right)^{-1}\right\| \leq C$. Since $I-K$ is injective, it follows from Theorem 4.3 that $(I-K)^{-1} \in$ $B(Y)$ with $\left\|(I-K)^{-1}\right\| \leq C$.

Theorem 4.4 is a generalization of a collectively compact operator approximation result of Anselone in [2, Theorem 1.6]. It reduces to a result in collectively compact operator theory when $X=Y$ for then all the topologies are the same, the operator convergence $K_{n} \stackrel{\beta}{\rightarrow} K$ is ordinary strong convergence, and we can take $S=\{I\}$ ( $I$ the identity operator). Theorem 4.4 is then still a generalization of $[\mathbf{2}$, Theorem 1.6], but reduces to it if we take $\mathcal{K}$ and $\mathcal{K}_{n}$ to each contain just a single operator. 
The following theorem, which will be used in the application in Section 5, is the special case of Theorems 4.1 and 4.4 which arises if we take $\mathcal{K}_{n}=\mathcal{K}$ for $n \in \mathbf{N}$.

Theorem 4.5. Suppose that $\mathcal{K} \subset \mathcal{L}(Y)$ is collectively sequentially compact with respect to the $\beta$ topology and $\beta$-sequentially compact. Suppose also that, for some sufficient set $S \subset i$ iso $(Y)$ and all $K \in \mathcal{K}$, $T K T^{-1} \in \mathcal{K}, T \in S$, and that $I-K$ is injective for all $K \in \mathcal{K}$. Then $(I-K)^{-1}$ is bounded for all $K \in \mathcal{K}$ as an operator on $(I-K)(Y)$ and

$$
\sup _{K \in \mathcal{K}}\left\|(I-K)^{-1}\right\|<\infty .
$$

Moreover, if, for every $K \in \mathcal{K}$ there exists $\left(K_{n}\right) \subset \mathcal{K}$ such that, for each $n$,

$$
I-K_{n} \text { injective } \Longrightarrow I-K_{n} \text { surjective }
$$

and $K_{n} \stackrel{\beta}{\rightarrow} K$, then $I-K$ is surjective for every $K \in \mathcal{K}$.

Remark 3.2. As an application of the point made in Remark 3.1, note that if Assumption A holds (as it does for all the examples considered in Section 2) then it follows from Corollaries 2.7 and 2.10 that $\mathcal{K} \subset \mathcal{L}(Y)$ is $\beta$-sequentially compact if and only if $\mathcal{K} \subset S(Y)$ and $\mathcal{K}$ is sequentially compact in the strong operator topology on $(Y, \beta)$. Further, and by Corollary 2.10, if $\mathcal{K} \subset \mathcal{L}(Y)$ is $\beta$-sequentially compact, then $K_{n} \stackrel{\beta}{\rightarrow} K$ if and only if $K_{n} \stackrel{S}{\rightarrow} K$. Thus, in the above theorem, if Assumption A holds then, without changing the content of the theorem, $K_{n} \stackrel{\beta}{\rightarrow} K$ can be replaced by $K_{n} \stackrel{S}{\rightarrow} K$ in the last line, and the condition that $K$ be $\beta$ sequentially compact be replaced by the conditions that $\mathcal{K} \subset S(Y)$ and is sequentially compact with respect to the strong operator topology on $(Y, \beta)$. These alternative conditions may be somewhat easier to check in applications.

Theorem 4.1, 4.4 and 4.5 can be combined with Theorem 4.3 to obtain a number of additional results. The following corollary of Theorems 4.1, 4.5 and 4.3 is perhaps of most significance.

Corollary 4.6. Suppose that the conditions of Theorem 4.1 are satisfied and that, additionally: 
(i) for every $n \in \mathbf{N}$ and $\tilde{K} \in \mathcal{K}_{n}$ it holds that

$$
I-\tilde{K} \text { injective } \Longrightarrow I-\tilde{K} \text { surjective; }
$$

(ii) for every $K \in \mathcal{K}$ there exists $\left(K_{n}\right) \in K^{\times}$such that $K_{n} \stackrel{\beta}{\rightarrow} K$.

Then the following statements hold.

(a) $N \in \mathbf{N}$ exists such that $I-\tilde{K}: Y \rightarrow Y$ is bijective for all $\tilde{K} \in \mathcal{K}_{n}$ and $n \geq N$, with

$$
C:=\sup _{\substack{n \geq N \\ \tilde{K} \in \mathcal{K}_{n}}}\left\|(I-\tilde{K})^{-1}\right\|<\infty .
$$

For $n \geq N$, equation (1.2) has a unique solution $\psi_{n} \in Y$ for every $\phi_{n} \in Y$ and $\left\|\psi_{n}\right\|_{\infty} \leq C\left\|\phi_{n}\right\|_{\infty}$.

(b) For all $K \in \mathcal{K}, I-K: Y \rightarrow Y$ is bijective so that $(I-K)^{-1}$ : $Y \rightarrow Y$ exists and is bounded. Further,

$$
\sup _{K \in \mathcal{K}}\left\|(I-K)^{-1}\right\| \leq C .
$$

Thus equation (1.1) has a unique solution $\psi \in Y$ for every $\phi \in Y$ and $\|\psi\|_{\infty} \leq C\|\phi\|_{\infty}$.

(c) If $K \in \mathcal{K}, K_{n} \in \mathcal{K}_{n}$, for $n=N, N+1, \ldots$, and $K_{n} \stackrel{\beta}{\rightarrow} K$, then $\left(I-K_{n}\right)^{-1} \stackrel{\beta}{\rightarrow}(I-K)^{-1}$. Thus, if $\phi \in Y, \phi_{n} \in Y$ for $n \geq N, \phi_{n} \stackrel{\beta}{\rightarrow} \phi$ and $\psi_{n}, \psi \in Y$ are the unique solutions of equations (1.2) and (1.1), respectively, it follows that $\psi_{n} \stackrel{\beta}{\rightarrow} \psi$.

5. Systems of integral equations on unbounded domains. As an application of the general results in the previous section which has important applications to problems of scattering by unbounded inhomogeneous layered media, as discussed in the introduction, we study in this section the solvability of the system of second kind integral equations:

$$
\psi_{i}=\phi_{i}+\sum_{j=1}^{N} K_{i j} \psi_{j}, \quad i=1, \ldots, N
$$


In this equation we suppose that $\phi_{i} \in Y_{i}:=B C\left(\bar{\Omega}_{i}\right)$, the set of bounded and continuous functions on $\bar{\Omega}_{i}$ is assumed known, $\psi_{i} \in Y_{i}$ is to be determined and $K_{i j}: Y_{j} \rightarrow Y_{i}$ is the integral operator defined by

$$
K_{i j} \psi(x)=\int_{\Omega_{j}} k_{i j}(x, y) \psi(y) d \mu_{j}(y), \quad x \in \bar{\Omega}_{i},
$$

$i, j=1, \ldots, N$. Further $\Omega_{j}$ is an open subset of $\mathbf{R}^{n_{j}}\left(n_{j} \geq 1\right)$ and $d \mu_{j}$ is $n_{j}$-dimensional Lebesgue measure. We note that $Y_{i}$ is a Banach space with the norm $\|\cdot\|_{\infty}^{(i)}$, defined by

$$
\|\phi\|_{\infty}^{(i)}:=\sup _{x \in \bar{\Omega}_{i}}|\phi(x)| .
$$

For some $M \in \mathbf{N}$, the functions $k_{i j}: \bar{\Omega}_{i} \times \Omega_{j} \rightarrow \mathbf{C}$ are assumed to take the form

$$
k_{i j}(x, y)=\sum_{m=1}^{M} k_{i j}^{(m)}(x, y) z_{j}^{(m)}(y)
$$

where $z_{j}^{(m)} \in Z_{j}:=L^{\infty}\left(\Omega_{j}\right)$ and $k_{i j}^{(m)}(x, \cdot) \in L^{1}\left(\Omega_{j}\right)$ for every $x \in \bar{\Omega}_{i}$ $(i, j=1, \ldots, N, m=1, \ldots, M)$.

We assume throughout this section that the following conditions on $k_{i j}^{(m)}$ and $\Omega_{j}$ hold, condition (C.1) a generalization of (1.6) and (1.7).

(C.1) $\sup _{x \in \bar{\Omega}_{i}} \int_{\Omega_{j}}\left|k_{i j}^{(m)}(x, y)\right| d \mu_{j}(y)<\infty$ and, for all $x \in \bar{\Omega}_{i}$,

$$
\int_{\Omega_{j}}\left|k_{i j}^{(m)}(x, y)-k_{i j}^{(m)}\left(x^{\prime}, y\right)\right| d \mu_{j}(y) \longrightarrow 0
$$

as $x^{\prime} \rightarrow x$ with $x^{\prime} \in \bar{\Omega}_{i}(i, j=1, \ldots, N, m=1, \ldots, M)$.

(C.2) For some $n_{0} \leq \min _{j} n_{j}$ and $i=1, \ldots, N$ there exists $a_{j}^{(i)} \in \mathbf{R}^{n_{i}}$, $j=1, \ldots, n_{0}$, and a bounded set $\omega_{i} \subset \Omega_{i}$ such that

(i) $\Omega_{i}=\omega_{i} \cup \bigcup_{P \in \mathbf{Z}^{n_{0}}} \omega_{i}^{(P)}$, where $\omega_{i}^{(P)}:=\omega_{i}+\sum_{j=1}^{n_{0}} a_{j}^{(i)} p_{j}$, for $P=\left(p_{1}, \ldots, p_{n_{0}}\right) \in \mathbf{Z}^{n_{0}}$;

(ii) $\omega_{i}^{(Q)} \cap \omega_{i}^{(P)}=\varnothing$ for $Q, P \in \mathbf{Z}^{n_{0}}, Q \neq P$;

(iii) $k_{i j}^{(m)}\left(x+a_{l}^{(i)}, y+a_{l}^{(j)}\right)=k_{i j}^{(m)}(x, y), x \in \bar{\Omega}_{i}, y \in \Omega_{j}, i, j=$ $1, \ldots, N, l=1, \ldots, n_{0}, m=1, \ldots, M$. 
Note that (i) and (ii) in (C.2) imply that $\Omega_{i}+a_{j}^{(i)}=\Omega_{j}, j=1, \ldots, n_{0}$, $i=1, \ldots, N$, so that (iii) makes sense. The point of (iii) is that it ensures a translation invariance property of the integral operators with kernels $k_{i j}^{(m)}$, expressed in (5.9) below.

Example 5.1. As perhaps the simplest example in which condition (C.2) is satisfied, consider the case when $M=N=1, \Omega_{1}=\mathbf{R}$, and, for some $a>0, k_{11}^{(1)}(x+a, y+a)=k_{11}^{(1)}(x, y), x, y \in \mathbf{R}$. Then (C.2) is satisfied with $n_{0}=1, a_{1}^{(1)}=a, \omega_{1}=[0, a)$. Note that $\omega_{1}^{(P)}=[P a,(P+1) a)$, for $P \in \mathbf{Z}^{n_{0}}=\mathbf{Z}$.

Example 5.2. As another example, suppose that $N \in \mathbf{N}, M=1$, and that, for some $n \in \mathbf{N}, \Omega_{j}=\mathbf{R}^{n}, j=1, \ldots, N$, and that for $i, j=1, \ldots, N, k_{i j}^{(1)}(x, y)=\kappa_{i j}(x-y), x, y \in \mathbf{R}^{n}$, with $\kappa_{i j} \in L^{1}\left(\mathbf{R}^{n}\right)$. Then the system of integral equations is, explicitly,

$$
\psi_{i}(x)=\phi_{i}(x)+\sum_{j=1}^{N} \int_{\mathbf{R}^{n}} \kappa_{i j}(x-y) z_{j}(y) \psi_{j}(y) d y, \quad x \in \mathbf{R}^{n},
$$

and $n_{j}=n, j=1, \ldots, N$. Let $\left\{e_{1}, \ldots, e_{n}\right\}$ be the usual orthonormal basis for $\mathbf{R}^{n}$ so that $x \in \mathbf{R}^{n}$ can be written as $x=\left(x_{1}, \ldots, x_{n}\right)=$ $x_{1} e_{1}+\cdots+x_{n} e_{n}$. Set $n_{0}=n, a_{j}^{(i)}=e_{j}, i=1, \ldots, N, j=1, \ldots, n$, and $\omega_{i}=[0,1)^{n}, i=1, \ldots, N$. Then, for $P=\left(p_{1}, \ldots, p_{n}\right) \in \mathbf{Z}^{n_{0}}=\mathbf{Z}^{n}$ and $i=1, \ldots, N$, it holds that $\omega_{i}^{(P)}=\left[p_{1}, p_{1}+1\right) \times \cdots \times\left[p_{n}, p_{n}+\right.$ 1) and (C.2) is satisfied. Assumption (C.1) is also satisfied, with $\sup _{x \in \bar{\Omega}_{i}} \int_{\Omega_{j}}\left|k_{i j}^{(1)}(x, y)\right| d \mu_{j}(y)=\left\|\kappa_{i j}\right\|_{L_{1}\left(\mathbf{R}^{n}\right)}, i, j=1, \ldots, N$. To see that (5.3) holds, note that it certainly holds for $k_{i j}^{(1)}(x, y)=\kappa_{i j}(x-y)$ if $\kappa_{i j} \in C_{0}^{\infty}\left(\mathbf{R}^{n}\right)$, the set of compactly supported infinitely differentiable functions on $\mathbf{R}^{n}$, and then use the density of $C_{0}^{\infty}\left(\mathbf{R}^{n}\right)$ in $L^{1}\left(\mathbf{R}^{n}\right)$ to see that it holds for all $\kappa_{i j} \in L^{1}\left(\mathbf{R}^{n}\right)$.

Example 5.3. [This example arises from the practical application of the theory of this section in [18], and a similar example, but with $N=4$, arises in [11].] Suppose that $N=2, \Omega_{1}=\mathbf{R}$, and that, for some $A>0, \Omega_{2}=\mathbf{R} \times(0, A)$. Then $n_{1}=1$ and $n_{2}=2$. Set $n_{0}=1$ and, for some $a>0$, set $a_{1}^{(1)}=a$ and $a_{1}^{(2)}=(a, 0)$. 
Suppose that (iii) in (C.2) holds with these choices of $a_{1}^{(1)}$ and $a_{1}^{(2)}$, i.e. that $k_{i j}^{(m)}\left(x+a_{1}^{(i)}, y+a_{1}^{(j)}\right)=k_{i j}^{(m)}(x, y), x \in \bar{\Omega}_{i}, y \in \Omega_{j}$, $i, j=1,2, m=1, \ldots, M$. Then (i) and (ii) in (C.2) also hold if we put $\omega_{1}=[0, a), \omega_{2}=[0, a) \times(0, A)$. Note also that, for $P \in \mathbf{Z}^{n_{0}}=\mathbf{Z}$, $\omega_{1}^{(P)}=[P a,(P+1) a)$ and $\omega_{2}^{(P)}=[P a,(P+1) a) \times(0, A)$.

Example 5.4. If $\Omega_{i}$ is bounded, then with the understanding that $\mathbf{Z}^{0}:=\varnothing$ and that (iii) is empty if $n_{0}=0,($ C.2) is trivially satisfied with $\omega_{i}=\Omega_{i}$ and $n_{0}=0$, and (iii) then imposes no restriction on the kernel $k_{i j}^{(m)}$.

Let $Z$ and $Y$ denote the product spaces $Z:=\prod_{j=1}^{N} Z_{j}$ and $Y:=$ $\prod_{j=1}^{N} Y_{j} \subset Z$. Let $\phi=\left(\phi_{1}, \ldots, \phi_{N}\right)^{t}, \psi=\left(\psi_{1}, \ldots, \psi_{N}\right)^{t} \in Y$, where $(\cdot, \ldots, \cdot)^{t}$ denotes the transpose of $(\cdot, \ldots, \cdot)$. For $m=1, \ldots, M$, define the matrix operator $K^{(m)}$ on $Z$ by

$$
K^{(m)}=\left(\begin{array}{ccc}
K_{11}^{(m)} & \cdots & K_{1 N}^{(m)} \\
& \ddots & \\
K_{N 1}^{(m)} & \cdots & K_{N N}^{(m)}
\end{array}\right)
$$

where $K_{i j}^{(m)}: Z_{j} \rightarrow Y_{i} \subset Z_{i}$ is the integral operator defined by (5.2) with $K_{i j}, k_{i j}$ replaced by $K_{i j}^{(m)}, k_{i j}^{(m)}$. For $z=\left(z_{1}, \ldots, z_{N}\right)^{t} \in Z$ define $\hat{z}$ by

$$
\hat{z}=\left(\begin{array}{ccc}
z_{1} & \cdots & 0 \\
& \ddots & \\
0 & \cdots & z_{N}
\end{array}\right)
$$

and, for $m=1, \ldots, M$ and $z \in Z$, define $K_{z}^{(m)}: Y \rightarrow Y$ by

$$
K_{z}^{(m)} \psi=K^{(m)}(\hat{z} \psi), \quad \psi \in Y .
$$

For $w=\left(w^{(1)}, \ldots, w^{(M)}\right) \in Z^{M}$ let $K_{w}$ denote the matrix integral operator

$$
K_{w}=\sum_{m=1}^{M} K_{w^{(m)}}^{(m)} .
$$


Then (5.1) can be abbreviated as

$$
\psi=\phi+K_{z} \psi,
$$

where $z=\left(z^{(1)}, \ldots, z^{(M)}\right)$ and $z^{(m)}=\left(z_{1}^{(m)}, \ldots, z_{N}^{(m)}\right)^{t}, m=$ $1, \ldots, M$.

Throughout, if (C.2) is satisfied, define $\tau:=\left\{\left(a_{l}^{(1)}, \ldots, a_{l}^{(N)}\right)^{t}: l=\right.$ $\left.1, \ldots, n_{0}\right\}$ and

$$
V:=\left\{\sum_{l=1}^{n_{0}} p_{l}\left(a_{l}^{(1)}, \ldots, a_{l}^{(N)}\right)^{t}: P=\left(p_{1}, \ldots, p_{n_{0}}\right) \in \mathbf{Z}^{n_{0}}\right\} .
$$

For $i=1, \ldots, N, P=\left(p_{1}, \ldots, p_{n_{0}}\right) \in \mathbf{Z}^{n_{0}}$ and $a_{i}=\sum_{l=1}^{n_{0}} p_{l} a_{l}^{(i)}$, define the translation operator $T_{a_{i}}: Z_{i} \rightarrow Z_{i}$ by

$$
T_{a_{i}} \psi(x)=\psi\left(x-a_{i}\right), \quad x \in \Omega_{i} .
$$

For $a=\left(a_{1}, \ldots, a_{N}\right) \in V$ define the matrix operator $T_{a}: Z \rightarrow Z$ by

$$
T_{a}=\left(\begin{array}{ccc}
T_{a_{1}} & & 0 \\
& \ddots & \\
0 & & T_{a_{N}}
\end{array}\right) .
$$

Then, if (C.2) is satisfied, it is easy to see that

$$
T_{a} K^{(m)}=K^{(m)} T_{a}, \quad a \in V, \quad m=1, \ldots, M,
$$

and that

$$
\forall x \in \Omega_{i} \exists a=\left(a_{1}, \ldots, a_{N}\right) \in V \text { such that } x-a_{i} \in \omega_{i},
$$

for $i=1, \ldots, N$.

Let $B(Y)$ denote the Banach space of bounded linear operators on $Y$ and $I$ the identity matrix operator on $Y$. The first result is an extension to a system of integral equations of the result for a single integral equation established in [10, Theorem 2.8]. We use the notion of weak* convergence in $Z^{M}$ extensively and pause to give a characterization of this convergence. Since $Z_{j}=L^{\infty}\left(\Omega_{j}\right)=\left(L^{1}\left(\Omega_{j}\right)\right)^{\prime}$, the dual space of 
$L^{1}\left(\Omega_{j}\right)$, it follows that $Z$ is the dual space of $L^{1}\left(\Omega_{1}\right) \times \cdots \times L^{1}\left(\Omega_{N}\right)$ and that $Z^{M}$ is the dual space of $\left(L^{1}\left(\Omega_{1}\right) \times \cdots \times L^{1}\left(\Omega_{N}\right)\right)^{M}$. As a consequence it follows that $\left(w_{n}\right)=\left(\left(w_{n}^{(1)}, \ldots, w_{n}^{(M)}\right)\right)$ is weak* convergent in $Z^{M}$ if and only if $\left(w_{n}^{(m)}\right)$ is weak* convergent in $Z$ for $m=1, \ldots, M$. In turn, $\left(w_{n}^{(m)}\right)=\left(w_{1 n}^{(m)}, \ldots, w_{N n}^{(m)}\right)^{t}$ is weak* convergent in $Z$ if and only if $\left(w_{j n}^{(m)}\right)$ is weak* convergent in $Z_{j}$ for $j=1, \ldots, N, m=1, \ldots, M$. Thus, and since $Z_{j}=\left(L^{1}\left(\Omega_{j}\right)\right)^{\prime},\left(w_{n}\right)$ is weak* convergent in $Z^{M}$ if and only if, for $j=1, \ldots, N, m=1, \ldots, M$ there exists $w_{j}^{(m)} \in Z_{j}$ such that

$$
\int_{\Omega_{j}} w_{j n}^{(m)} \varphi d \mu_{j} \longrightarrow \int_{\Omega_{j}} w_{j}^{(m)} \varphi d \mu_{j}
$$

as $n \rightarrow \infty$, for all $\varphi \in L^{1}\left(\Omega_{j}\right)$. It may be helpful to note that (5.11) holds for all $\varphi \in L^{1}\left(\Omega_{j}\right)$ if and only if

$$
\sup _{n}\left\|w_{j n}^{(m)}\right\|_{\infty}<\infty, \int_{\Omega_{j}} w_{j n}^{(m)} \varphi d \mu_{j} \longrightarrow \int_{\Omega_{j}} w_{j}^{(m)} \varphi d \mu_{j}, \forall \varphi \in C_{0}^{\infty}\left(\Omega_{j}\right),
$$

where $C_{0}^{\infty}\left(\Omega_{j}\right)$ denotes the set of $C^{\infty}$ compactly supported functions with support in $\Omega_{j}$. We will say that $W \subset \mathbf{Z}^{M}$ is weak* sequentially compact if every sequence in $W$ has a subsequence converging weak* to some $w \in W$.

Theorem 5.1. Suppose that (C.1) and (C.2) are satisfied, that $W \subset$ $Z^{M}$ is weak ${ }^{*}$ sequentially compact, that $T_{a}(W):=\left\{\left(T_{a} z^{(1)}, \ldots, T_{a} z^{(M)}\right)\right.$ : $\left.\left(z^{(1)}, \ldots, z^{(M)}\right) \in W\right\}=W, a \in \tau$, and that $I-K_{z}$ is injective for all $z \in W$. Then $\left(I-K_{z}\right)^{-1}$ exists as an operator on the range space $\left(I-K_{z}\right)(Y)$ for all $z \in W$ and

$$
\sup _{z \in W}\left\|\left(I-K_{z}\right)^{-1}\right\|<\infty .
$$

If also, for every $z \in W$, there exists a sequence $\left(z_{n}\right) \subset W$ such that $\left(z_{n}\right)$ converges weak $k^{*}$ to $z$ in $Z^{M}$ and for all $n$,

$$
I-K_{z_{n}} \text { injective } \Longrightarrow I-K_{z_{n}} \text { surjective, }
$$

then also $I-K_{z}$ is surjective for each $z \in W$ so that $\left(I-K_{z}\right)^{-1} \in B(Y)$. 
To apply Theorem 4.5 to prove Theorem 5.1 let $X_{j}=C\left(\Omega_{j}\right)$, a Fréchet space with the semi-norms $|\cdot|_{n}^{(j)}, n \in \mathbf{N}$, defined by

$$
|\phi|_{n}^{(j)}:=\sup _{\substack{x \in \Omega_{j} \\|x| \leq n}}|\phi(x)|, n \in \mathbf{N}, \phi \in C\left(\Omega_{j}\right), j=1, \ldots, N .
$$

Then for $j=1, \ldots, N$, the subspace $\left\{\phi \in X_{j}: \sup _{n \in \mathbf{N}}|\phi|_{n}^{(j)}<\infty\right\}$ of $X_{j}$ is the Banach space $Y_{j}=B C\left(\Omega_{j}\right)$. In each closed subspace $Y_{j} \subset X_{j}$ we introduce convergence in the strict topology on $Y_{j}$ of Buck [7]. For $\left(\phi_{n}\right) \subset Y_{j}, \phi \in Y_{j}$, we write $\phi_{n} \stackrel{s}{\rightarrow} \phi$ if $\phi_{n}$ converges to $\phi$ in this topology. Buck [7] shows that $\phi_{n} \stackrel{s}{\rightarrow} \phi$ if and only if $\sup _{n \in \mathbf{N}}\left\|\phi_{n}\right\|_{\infty}^{(j)}<\infty$ and $\phi_{n}(x) \rightarrow \phi(x)$ uniformly on every compact subset of $\bar{\Omega}_{j}$. Let $X$ denote the product space $X:=\prod_{j=1}^{N} X_{j}$, a Fréchet space with the semi-norms $|\cdot|_{n}$ defined by $|\phi|_{n}:=\max _{1 \leq j \leq N}\left|\phi_{j}\right|_{n}^{(j)}$, $\phi=\left(\phi_{1}, \ldots, \phi_{N}\right)^{t} \in X$. Then the Banach space $Y$, defined by $(2.1)$, is the subspace of $X$,

$$
\left\{\phi=\left(\phi_{1}, \ldots, \phi_{N}\right)^{t} \in X:\|\phi\|_{\infty}:=\sup _{n \in \mathbf{N}}|\phi|_{n}<\infty\right\}
$$

which, since $\|\phi\|_{\infty}=\sup _{n \in \mathbf{N}}|\phi|_{n}=\max _{1 \leq j \leq N}\left\|\phi_{j}\right\|_{\infty}^{(j)}$, is precisely the product space $\prod_{j=1}^{N} Y_{j}$.

It is easy to see, using Theorem 2.1(iv), that sequential convergence in the $\beta$ topology on $Y$ of Section 2 has the following characterization: for $\phi_{n}=\left(\phi_{1 n}, \ldots, \phi_{N n}\right)^{t} \in Y$ and $\phi=\left(\phi_{1}, \ldots, \phi_{N}\right)^{t} \in Y$,

$$
\phi_{n} \stackrel{\beta}{\longrightarrow} \phi \text { iff } \phi_{j n} \stackrel{s}{\rightarrow} \phi_{j}, \quad j=1, \ldots, N
$$

For $W \subset Z^{M}$, let us define the set of integral operators, $\mathcal{K}_{W}$, by

$$
\mathcal{K}_{W}:=\left\{K_{w}: w \in W\right\} \subset \mathcal{L}(Y)
$$

Lemma 5.2. Suppose that (C.1) is satisfied and that $W \subset Z^{M}$ is bounded. Then $\mathcal{K}_{W}$ is collectively sequentially compact with respect to the $\beta$ topology on $Y$. 
Proof. Let $B \subset Y$ be bounded and let $\left(\phi_{n}\right) \subset \cup_{w \in W} K_{w} B$. We will prove that $\left(\phi_{n}\right)$ has a $\beta$-convergent subsequence. By [10, Lemma 2.2] there exists a subsequence of $\left(\phi_{n}\right)$ such that each component of the subsequence is strictly convergent. Applying (5.12), the subsequence is $\beta$-convergent. The lemma is thus proved.

Lemma 5.3. Suppose that (C.1) is satisfied and that $\left(w_{n}\right) \subset Z^{M}$, $w \in Z^{M}$ and $w_{n} \stackrel{w *}{\rightarrow} w$. Then $K_{w_{n}} \stackrel{\beta}{\rightarrow} K_{w}$.

Proof. Let $\phi_{n}=\left(\phi_{1 n}, \ldots, \phi_{N_{n}}\right)^{t} \in Y, \phi=\left(\phi_{1}, \ldots, \phi_{N}\right)^{t} \in Y$ be such that $\phi_{n} \stackrel{\beta}{\rightarrow} \phi$, that is, $\phi_{j n} \stackrel{s}{\rightarrow} \phi_{j}, j=1, \ldots, N$. We want to prove that $K_{w_{n}} \phi_{n} \stackrel{\beta}{\rightarrow} K_{w} \phi$ as $n \rightarrow \infty$. Let $w_{n}=\left(w_{n}^{(1)}, \ldots, w_{n}^{(M)}\right) \in W$ and $w=$ $\left(w^{(1)}, \ldots, w^{(M)}\right) \in W$. From the definition (5.4)-(5.6) we see that the $i$ th components of $K_{w_{n}} \phi_{n}$ and $K_{w} \phi$ are $\sum_{m=1}^{M} \sum_{j=1}^{N} K_{i j}^{(m)}\left(w_{i n}^{(m)} \phi_{i n}\right)$ and $\sum_{m=1}^{M} \sum_{j=1}^{N} K_{i j}^{(m)}\left(w_{i}^{(m)} \phi_{i}\right)$, respectively, so that, in view of (5.12), it is sufficient to prove that, for each $i, j, m, K_{i j}^{(m)}\left(w_{i n}^{(m)} \phi_{i n}\right) \stackrel{s}{\rightarrow}$ $K_{i j}^{(m)}\left(w_{i}^{(m)} \phi_{i}\right)$. But this follows from [10, Corollary 2.3] since the kernel of each $K_{i j}^{(m)}$ satisfies (C.1) and it is easy to see from the fact that $w_{i n}^{(m)} \stackrel{w *}{\rightarrow} w_{i}^{(m)}$ and $\phi_{i n} \stackrel{s}{\rightarrow} \phi_{i}$ that $w_{i n}^{(m)} \phi_{i n} \stackrel{w *}{\rightarrow} w_{i}^{(m)} \phi_{i}$.

The above lemma has the following two useful corollaries.

Corollary 5.4. Suppose that (C.1) is satisfied and that $w \in Z^{M}$, $\left(z_{n}\right) \subset Z, z \in Z$ and $z_{n} \stackrel{w *}{\rightarrow} z$. Then $K_{w} z_{n} \stackrel{\beta}{\rightarrow} K_{w} z$.

Corollary 5.5. Suppose that (C.1) is satisfied and that $W \subset Z^{m}$ is weak* sequentially compact. Then for every sequence $\left(K_{n}\right) \subset \mathcal{K}_{W}$ there exists a subsequence $\left(K_{n_{m}}\right)$ and $K \in \mathcal{K}_{W}$ such that $K_{n_{m}} \stackrel{\beta}{\rightarrow} K$.

Set $S=\left\{T_{a}: a \in V\right\}$. We then have the following result.

Lemma 5.6. Suppose that (C.2) is satisfied. Then $S \subset$ iso $(Y)$ is sufficient. 
Proof. Since each $\omega_{i}$ is bounded, we can choose an integer $J \geq 1$ such that, for $i=1, \ldots, N$ and $x \in \omega_{i}$, it holds that $|x| \leq J$. Let $\phi=\left(\phi_{1}, \ldots, \phi_{N}\right)^{t} \in Y$. Then there exists $i \in\{1, \ldots, N\}$ and $x_{i} \in \Omega_{i}$ such that $\left|\phi_{i}\left(x_{i}\right)\right| \geq(1 / 2)\|\phi\|_{\infty}$. By (5.10) there is an $a=\left(a_{1}, \ldots, a_{N}\right) \in V$ such that $x_{i}-a_{i} \in \omega_{i} \subset \Omega_{i}$. Thus $\left|T_{-a_{i}} \phi_{i}\right|_{J}^{(i)} \geq\left|\left(T_{-a_{i}} \phi_{i}\right)\left(x_{i}-a_{i}\right)\right|=\left|\phi_{i}\left(x_{i}\right)\right| \geq(1 / 2)\|\phi\|_{\infty}$. Letting $\tilde{a}=\left(-a_{1}, \ldots,-a_{N}\right)$ we have that $\tilde{a} \in V, T_{\tilde{a}} \in S$ and

$$
\left|T_{\tilde{a}} \phi\right|_{J}=\max _{1 \leq j \leq N}\left|T_{-a_{j}} \phi_{j}\right|_{J}^{(j)} \geq \frac{1}{2}\|\phi\|_{\infty},
$$

which completes the proof of the lemma.

With the help of Lemmas 5.2-5.6 and Corollary 5.5 we can now prove Theorem 5.1 making use of Theorem 4.5.

Proof of Theorem 5.1. To make use of Theorem 4.5 to prove Theorem 5.1, let $\mathcal{K}=\mathcal{K}_{W}$ and $S=\left\{T_{a}: a \in V\right\}$. Then $I-K$ is injective for all $K \in \mathcal{K}$ and, by (5.9), (5.4)-(5.6), and the fact that $T_{a}(W)=W$, $a \in \tau$, it follows that, for all $K \in \mathcal{K}$ and $T \in S, T K T^{-1} \in \mathcal{K}$. Thus by Lemmas 5.2 and 5.6 and Corollary 5.5 , all the assumptions in the first part of Theorem 4.5 have been verified. To verify the assumptions in the second part of Theorem 4.5, let $K=K_{z} \in \mathcal{K}$ for some $z \in W$. Then by the hypothesis in the second part of Theorem 5.1, a sequence $\left(z_{n}\right) \subset W$ exists such that $z_{n} \stackrel{w^{*}}{\rightarrow} z$ and $I-K_{z_{n}}$ injective implies $I-K_{z_{n}}$ surjective for all $n$. Set $K_{n}=K_{z_{n}}$. It follows from Lemma 5.3 that $K_{n} \stackrel{\beta}{\rightarrow} K$. Thus all the assumptions in Theorem 4.5 have been verified, and Theorem 5.1 follows from Theorem 4.5.

The last part of Theorem 5.1 becomes useful with the addition of conditions on $k_{i j}^{(m)}$ and $z_{j}^{(m)}$ which ensure that $I-K_{z}$ injective $\Rightarrow I-K_{z}$ surjective. The next theorem provides such conditions and, at the same time, illustrates the application of the first part of Theorem 5.1.

Theorem 5.7. If (C.1) and (C.2) are satisfied, $z=\left(\left(z_{1}^{(1)}, \ldots, z_{N}^{(1)}\right)^{t}\right.$, $\left.\ldots,\left(z_{1}^{(M)}, \ldots, z_{N}^{(M)}\right)^{t}\right) \in Z^{M}$ and, for some constants $\lambda_{j}^{(m)} \in \mathbf{C}$, 
$j=1, \ldots, N, m=1, \ldots, M$, it holds that

$$
\text { ess. } \sup _{|x| \geq A, x \in \Omega_{j}}\left|z_{j}^{(m)}(x)-\lambda_{j}^{(m)}\right| \longrightarrow 0
$$

as $A \rightarrow \infty$, then

$I-K_{\lambda}, I-K_{z}$ injective $\Longrightarrow I-K_{z}$ surjective, $\left(I-K_{z}\right)^{-1} \in B(Y)$,

where $\lambda=\left(\left(\lambda_{1}^{(1)}, \ldots, \lambda_{N}^{(1)}\right)^{t}, \ldots,\left(\lambda_{1}^{(M)}, \ldots, \lambda_{N}^{(M)}\right)^{t}\right)$.

To prove this theorem we first study the invertibility of $I-K_{z}$ in subspaces of $Y$ consisting of functions periodic on $\Omega_{i}, i=1, \ldots, N$.

Suppose that (C.2) is satisfied and, for $l \in \mathbf{N}$, let $V^{(l)}:=\left\{3^{l-1} a\right.$ : $\left.a=\left(a_{1}, \ldots, a_{N}\right) \in V\right\}$, so that $V^{(l)} \subseteq V$ with equality if $l=1$, and define the closed subspaces $Z^{(l)}, Y^{(l)} \subset Z$, by $Z^{(l)}:=\left\{\psi \in Z: T_{a} \psi=\right.$ $\left.\psi, a \in V^{(l)}\right\}$ and $Y^{(l)}:=Y \cap Z^{(l)}$. Clearly, $\psi=\left(\psi_{1}, \ldots, \psi_{N}\right) \in Z^{(l)}$ if and only if $\psi \in Z$ and, for every $a=\left(a_{1}, \ldots, a_{N}\right) \in V^{(l)}$, it holds that $\psi_{i}\left(x+a_{i}\right)=\psi_{i}(x), x \in \bar{\Omega}_{i}, i=1, \ldots, N$. If $V=\varnothing$ (the case if each $\Omega_{i}$ is bounded) then $Z^{(l)}=Z$.

For $P=\left(p_{1}, \ldots, p_{n_{0}}\right) \in \mathbf{Z}^{n_{0}}$, define $|P|:=\max \left(\left|p_{1}\right|, \ldots,\left|p_{n_{0}}\right|\right)$. If (C.2) is satisfied, for $i=1, \ldots, N$, let $\omega_{i 1}:=\omega_{i}$ and define, for $l=2,3, \ldots$,

$$
\omega_{i l}:=\bigcup_{\substack{P \in \mathbf{Z}^{n_{0}} \\|P| \leq\left(3^{l-1}-1\right) / 2}} \omega_{i}^{(P)} .
$$

For $P \in \mathbf{Z}^{n_{0}}$ and $l \in \mathbf{N}$, define

$$
\omega_{i l}^{(P)}:=\omega_{i l}+\sum_{j=1}^{n_{0}} 3^{l-1} a_{j}^{(i)} p_{j}
$$

and note that, by (C.2), $\Omega_{i}=\omega_{i l} \cup\left(\bigcup_{P \in \mathbf{Z}^{n_{0}}} \omega_{i l}^{(P)}\right)$ and $\omega_{i l}^{(P)} \cap \omega_{i l}^{(Q)}=\varnothing$ for $P, Q \in \mathbf{Z}^{n_{0}}, P \neq Q(i=1, \ldots, N)$. For $l \in \mathbf{N}$ and $i=1, \ldots, N$, define the periodic extension operator $P_{i}^{(l)}: Z_{i} \rightarrow Z_{i}$ by

$$
P_{i}^{(l)} \psi(x):=\psi\left(x-\sum_{j=1}^{n_{0}} 3^{l-1} a_{j}^{(i)} p_{j}\right), \quad x \in \omega_{i l}^{(P)},
$$


for $P=\left(p_{1}, \ldots, p_{n_{0}}\right) \in \mathbf{Z}^{n_{0}}$ and the matrix operator $P^{(l)}: Z \rightarrow Z^{(l)}$ by

$$
P^{(l)}=\left(\begin{array}{ccc}
P_{1}^{(l)} & & 0 \\
& \ddots & \\
0 & & P_{N}^{(l)}
\end{array}\right) .
$$

Lemma 5.8. If (C.1)-(C.2) are satisfied and $z=\left(\left(\lambda_{1}^{(1)}, \ldots, \lambda_{N}^{(1)}\right)^{t}\right.$, $\left.\ldots,\left(\lambda_{1}^{(M)}, \ldots, \lambda_{N}^{(M)}\right)^{t}\right)$ with some constants $\lambda_{j}^{(m)} \in \mathbf{C}$, then for $l \in \mathbf{N}$, $K_{z}: Z^{(l)} \rightarrow Y^{(l)}$ and is compact.

Proof. Condition (C.1) ensures that $K_{z}: Z \rightarrow Y$ and is bounded. If (C.2) additionally holds, then $K_{z}: Z^{(l)} \rightarrow Y^{(l)}$ and is bounded. Further, since by the Banach-Alaoglu theorem the unit ball in $Z$ is weak* sequentially compact, it follows from Corollary 5.4 that the image of every bounded sequence in $Z$ has a $\beta$-convergent subsequence. That $K_{z}: Z^{(l)} \rightarrow Y^{(l)}$ is compact now follows from the fact, clear from the characterization (5.12), that every $\beta$-convergent sequence in $Y^{(l)} \subset Y$ is norm convergent.

The following lemma extends [10, Theorem 2.10], which deals with a single integral equation, to the case of a system of integral equations, and explores the invertibility of $I-K_{z}$ for the special case when $z=$ $\left(\left(\lambda_{1}^{(1)}, \ldots, \lambda_{N}^{(1)}\right)^{t}, \ldots,\left(\lambda_{1}^{(M)}, \ldots, \lambda_{N}^{(M)}\right)^{t}\right)$ with some constants $\lambda_{j}^{(m)} \in$ C.

Lemma 5.9. If (C.1)-(C.2) are satisfied and $z=\left(\left(\lambda_{1}^{(1)}, \ldots, \lambda_{N}^{(1)}\right)^{t}\right.$, $\left.\ldots,\left(\lambda_{1}^{(M)}, \ldots, \lambda_{N}^{(M)}\right)^{t}\right)$ with some constants $\lambda_{j}^{(m)} \in \mathbf{C}$, then

$$
I-K_{z} \text { injective } \Longrightarrow I-K_{z} \text { surjective, }\left(I-K_{z}\right)^{-1} \in B(Y) .
$$

Proof. We have that, for $l \in \mathbf{N}$,

$$
\begin{aligned}
I-K_{z} \text { injective on } Y & \Longrightarrow I-K_{z} \text { injective on } Y^{(l)} \\
& \Longrightarrow I-K_{z} \text { surjective on } Y^{(l)},
\end{aligned}
$$


since, by Lemma 5.8, $K_{z}$ is compact on $Y^{(l)}$. Thus, if $I-K_{z}$ is injective (on $Y$ ), then $Y^{(l)} \subset\left(I-K_{z}\right)(Y)$ for every $l \in \mathbf{N}$.

Let $W=\{z\}$. If $I-K_{z}$ is injective then $W$ satisfies the conditions of the first part of Theorem 5.1, so that $\left(I-K_{z}\right)^{-1}$ exists as an operator on the range space $\left(I-K_{z}\right)(Y)$ and $\left(I-K_{z}\right)^{-1}$ is bounded.

So suppose that $I-K_{z}$ is injective, let $\phi \in Y$, note that $K_{z} P^{(n)} \phi \in$ $Y^{(n)} \subset\left(I-K_{z}\right)(Y)$ by the above and Lemma 5.8, and define $\psi^{(n)}:=$ $\phi+\left(I-K_{z}\right)^{-1} K_{z} P^{(n)} \phi$, for $n \in \mathbf{N}$. Then $\left(\psi^{(n)}\right)$ is bounded and $\psi^{(n)}=K_{z} \psi^{(n)}+\phi^{(n)}$, where $\phi^{(n)}:=\phi+K_{z}\left(P^{(n)} \phi-\phi\right)$. Since $P^{(n)} \phi \stackrel{w^{*}}{\rightarrow} \phi$ it follows from Corollary 5.4 that $K_{z}\left(P^{(n)} \phi-\phi\right) \stackrel{\beta}{\rightarrow} 0$, so that $\phi^{(n)} \stackrel{\beta}{\rightarrow} \phi$. It follows from Lemmas 5.2, 5.3 and 4.2 that $\phi \in\left(I-K_{z}\right)(Y)$. Thus $I-K_{z}$ is surjective and $\left(I-K_{z}\right)^{-1} \in B(Y)$.

Proof of Theorem 5.7. We write $I-K_{z}=I-K_{\lambda}-K_{z-\lambda}$. The kernel of each component of the matrix operator $K_{z-\lambda}$ satisfies the conditions (C.1) and (C.2) and further, by the assumptions of the theorem, condition $\mathrm{C}$ of $[\mathbf{9}]$ is satisfied (that $\int_{\Omega_{j}}\left|\kappa_{i j}(x, y)\right| d \mu_{j}(y) \rightarrow 0$ as $|x| \rightarrow \infty$ with $\left.x \in \bar{\Omega}_{i}\right)$. Thus by [9, Lemma 2.1] each component of the matrix operator $K_{z-\lambda}$ is compact so that $K_{z-\lambda}$ is compact. (Note that both Condition $\mathrm{C}$ and Lemma 2.1 in [9] are for the case when $\Omega_{i}=\Omega_{j}$. However, a version of Lemma 2.1 for the case when $\Omega_{i} \neq \Omega_{j}$ can be proved using exactly the same argument as for the case when $\Omega_{i}=\Omega_{j}$.) By Lemma 5.9, $\left(I-K_{\lambda}\right)^{-1} \in B(Y)$ so that $I-K_{\lambda}$ is Fredholm of index zero. Since $K_{z-\lambda}$ is compact, $I-K_{z}$ is also Fredholm of index zero and the result follows.

Theorems 5.1 and 5.7 are both applied in references [18] and [11] to study, respectively, coupled $2 \times 2$ and $4 \times 4$ systems of boundary and domain integral equations, modeling wave scattering problems by rough interfaces and inhomogeneous layers.

Acknowledgments. This work was supported by the UK Engineering and Physical Sciences Research Council under grants GR/M59433 and GR/N14415. 


\section{APPENDIX}

This appendix contains certain rather technical proofs, deferred from Section 3, in particular the proofs of Theorems 3.7 and 3.8.

Lemma A.1. Suppose that Assumption A holds, $\varepsilon>0$ and $m \in \mathbf{N}$. Then there exists $n>m$ and $P_{m}: Y \rightarrow Y_{m}$ such that, for $\psi \in Y$, $\chi \in Y,|\chi|_{n}^{\prime} \leq \varepsilon$.

$$
\left\|\psi-P_{m} \psi+\chi\right\|_{\infty} \leq \max \left(\|\psi\|_{\infty},\|\chi\|_{\infty}\right)+2 \varepsilon .
$$

Proof. By Assumption A there exists $P_{m}: Y \rightarrow Y_{m}$ and $l>m$ such that

$$
\left\|\psi-P_{m} \psi-\phi\right\|_{\infty} \leq \max \left(\|\psi\|_{\infty},\|\phi\|_{\infty}\right), \quad \psi \in Y, \phi \in Y_{l} .
$$

Noting that Assumption A implies Assumption $\mathrm{A}^{\prime}$, there exists $n>l$ and $P_{l}: Y \rightarrow Y_{l}$ such that

$$
\left\|\chi-P_{l} \chi\right\|_{\infty} \leq|\chi|_{n}^{\prime}, \quad \chi \in Y .
$$

Thus, for $\psi \in Y, \chi \in Y,|\chi|_{n}^{\prime} \leq \varepsilon$,

$$
\begin{aligned}
\left\|\psi-P_{m} \psi+\chi\right\|_{\infty} & \leq\left\|\psi-P_{m} \psi+P_{l} \chi\right\|_{\infty}+\left\|\chi-P_{l} \chi\right\|_{\infty} \\
& \leq \max \left(\|\psi\|_{\infty},\left\|P_{l} \chi\right\|_{\infty}\right)+|\chi|_{n}^{\prime} \\
& \leq \max \left(\|\psi\|_{\infty},\|\chi\|_{\infty}+\left\|\chi-P_{l} \chi\right\|_{\infty}\right)+\varepsilon \\
& \leq \max \left(\|\psi\|_{\infty},\|\chi\|_{\infty}\right)+2 \varepsilon
\end{aligned}
$$

Lemma A.2. Suppose that Assumption A holds and that $L \in S(Y)$, $m \in \mathbf{N}, \psi \in Y$. Then, for all $\eta>0$, there exists $n>m, \varepsilon>0$ and $\psi^{\prime} \in Y$ such that

$$
\left|\psi-\psi^{\prime}\right|_{m}^{\prime}=0, \quad\left|L\left(\psi-\psi^{\prime}\right)\right|_{m}^{\prime} \leq \eta,
$$

and such that, for all $\chi \in Y$ with $|\chi|_{n}^{\prime} \leq \varepsilon$,

$$
\left\|\psi^{\prime}+\chi\right\|_{\infty} \leq \max \left(\|\psi\|_{\infty},\|\chi\|_{\infty}\right)+\eta .
$$


Proof. For $j \in \mathbf{N}$ choose $\varepsilon_{j}>0$ such that $\varepsilon_{j} \rightarrow 0$ as $j \rightarrow \infty$. From Lemma A.1 it follows, that for every $j \in \mathbf{N}$, we can find $n_{j} \in \mathbf{N}$ with $n_{j}>m+j$, and $\phi_{j} \in Y_{m+j}$, such that

$$
M_{j}:=\sup _{\substack{\chi \in Y \\|\chi|_{n_{j}} \leq \varepsilon_{j}}}\left[\left\|\psi-\phi_{j}+\chi\right\|_{\infty}-\max \left(\|\psi\|_{\infty},\|\chi\|_{\infty}\right)\right] \leq 2 \varepsilon_{j} \longrightarrow 0
$$

as $j \rightarrow \infty$. Since $M_{j} \geq\left\|\psi-\phi_{j}\right\|_{\infty}-\|\psi\|_{\infty} \geq\left\|\phi_{j}\right\|_{\infty}-2\|\psi\|_{\infty}$, it follows, applying Theorem 2.1 (iv) that $\phi_{j} \stackrel{\beta}{\rightarrow} 0$ and, since $L \in S(Y)$, that $L \phi_{j} \stackrel{\beta}{\rightarrow} 0$. Thus there exists $J \in \mathbf{N}$ such that $\phi_{J} \in Y_{m},\left|L \phi_{J}\right|_{m}^{\prime} \leq \eta$, $M_{J} \leq \eta$. Now set $\psi^{\prime}=\psi-\phi_{J}, n=n_{J}, \varepsilon=\varepsilon_{J}$. Then these choices satisfy all the conditions of the lemma.

Theorem A.3. Suppose Assumption A holds and $K_{n} \in S(Y)$, $n \in \mathbf{N}$. Then $K_{n} \stackrel{S}{\rightarrow} 0$ if and only if $K_{n} \stackrel{\beta}{\rightarrow} 0$.

Proof. Suppose that this is false. Then, by Lemma 3.4 there exists $\varepsilon>0, m \in \mathbf{N}$ and sequences $\left(K_{n}\right) \subset S(Y)$ and $\left(\psi_{n}\right) \subset Y$, with $\psi_{n} \stackrel{\beta}{\rightarrow} 0$ and $K_{n} \stackrel{S}{\rightarrow} 0$ such that

$$
\left|K_{n} \psi_{n}\right|_{m}^{\prime} \geq \varepsilon
$$

Our method of proof will be to construct approximations $\tilde{\psi}_{n}$ to the members of some finite or infinite subsequence of $\left(\psi_{n}\right)$ in such a way that

$$
\phi=\sum_{n} \tilde{\psi}_{n}
$$

exists and satisfies, for some sequence $\left(N_{n}\right) \subset \mathbf{N}$, with $N_{n} \rightarrow \infty$ as $n \rightarrow \infty$,

$$
\left|K_{N_{n}} \phi\right|_{m}^{\prime} \geq \frac{\varepsilon}{3}, \quad n \in \mathbf{N}
$$

This will contradict $K_{n} \stackrel{S}{\rightarrow} 0$.

As the first stage we show, by induction, the existence of sequences $\left(\phi_{j}\right) \subset Y$, each $\phi_{j}$ a partial sum of $($ A. 1$),\left(\varepsilon_{j}\right) \subset(0, \infty)$ and strictly 
monotonic increasing sequences $\left(N_{j}\right) \subset \mathbf{N},\left(n_{j}\right) \subset \mathbf{N}$ such that, for $j=1,2, \ldots$, it holds that

$$
\begin{aligned}
\left|K_{N_{n}} \phi_{j}\right|_{m}^{\prime}>\frac{\varepsilon}{3}, \quad n & =1,2, \ldots, j, \\
\left|\phi_{j+1}-\phi_{j}\right|_{n_{j}}^{\prime} & \leq 2^{-j}
\end{aligned}
$$

and

(A.4) $\chi \in Y,|\chi|_{n_{j}}^{\prime} \leq \varepsilon_{j} \Longrightarrow\left\|\phi_{j}+\chi\right\|_{\infty} \leq \max \left(M,\|\chi\|_{\infty}\right)+2-2^{1-j}$,

where $M:=\sup _{n \in \mathbf{N}}\left\|\psi_{n}\right\|_{\infty}$.

Note first that, by Lemma A.2, we can choose $\phi_{1} \in Y$ (an approximation to $\left.\psi_{1}\right) n_{1} \in \mathbf{N}$ and $\varepsilon_{1}>0$ such that

$$
\left|K_{1} \phi_{1}\right|_{m}^{\prime} \geq \varepsilon-\left|K_{1}\left(\psi_{1}-\phi_{1}\right)\right|_{m}^{\prime}>\frac{\varepsilon}{3}
$$

and such that

$$
\left\|\phi_{1}+\chi\right\|_{\infty} \leq \max \left(\left\|\psi_{1}\right\|_{\infty},\|\chi\|_{\infty}\right)+1,
$$

for all $\chi \in Y$ with $|\chi|_{n_{1}}^{\prime} \leq \varepsilon_{1}$. Then (A.2) and (A.4) hold with $j=1$ and $N_{1}=1$.

Given $j \in \mathbf{N}, N_{1}<N_{2}<\cdots<N_{j}, n_{j} \in \mathbf{N}, \varepsilon_{j}>0$ and $\phi_{j} \in Y$ which satisfies (A.2) and (A.4), define $\phi_{j+1}:=\phi_{j}, N_{j+1}:=N, n_{j+1}:=n_{j}+1$, $\varepsilon_{j+1}:=\varepsilon_{j}$, in the case that

$$
\left|K_{N} \phi_{j}\right|_{m}^{\prime}>\varepsilon / 3
$$

for some $N>N_{j}$. Then $N_{j+1}>N_{j}$, (A.3) holds, and (A.2) and (A.4) hold with $j$ replaced by $j+1$. Consider now the case that (A.5) does not hold for any $N>N_{j}$. Since $\psi_{n} \stackrel{\beta}{\rightarrow} 0$ and $K_{l} \in S(Y), l \in \mathbf{N}$, so that also $K_{l} \psi_{n} \stackrel{\beta}{\rightarrow} 0$, we can choose $N_{j+1}>N_{j}$ such that

$$
\left|\psi_{N_{j+1}}\right|_{n_{j}}^{\prime} \leq \min \left[\frac{\varepsilon_{j}}{2}, 2^{-j}\right], \quad\left|K_{N_{l}} \psi_{N_{j+1}}\right|_{m}^{\prime}<\frac{\eta}{2}, \quad l=1, \ldots, j,
$$

where

$$
\eta:=\min _{l=1, \ldots, j}\left|K_{N_{l}} \phi_{j}\right|_{m}^{\prime}-\frac{\varepsilon}{3}>0 .
$$


Then, by Lemma A.2, it is possible to choose $\tilde{\psi}_{j+1} \in Y, n_{j+1}>n_{j}$ and $\varepsilon_{j+1} \in\left(0, \varepsilon_{j} / 2\right)$ such that

$$
\begin{gathered}
\left|\psi_{N_{j+1}}-\tilde{\psi}_{j+1}\right|_{n_{j}}^{\prime}=0, \\
\left|K_{N_{l}}\left(\psi_{N_{j+1}}-\tilde{\psi}_{j+1}\right)\right|_{m}^{\prime}<\min \left[\frac{\varepsilon}{3}, \frac{\eta}{2}\right], \\
l=1, \ldots, j+1,
\end{gathered}
$$

and such that, for all $\chi \in Y$ with $|\chi|_{n_{j+1}}^{\prime} \leq \varepsilon_{j+1}$,

$$
\left\|\tilde{\psi}_{j+1}+\chi\right\|_{\infty} \leq \max \left(\left\|\psi_{N_{j+1}}\right\|_{\infty},\|\chi\|_{\infty}\right)+2^{-j} .
$$

It further holds that

$$
\left|\tilde{\psi}_{j+1}\right|_{n_{j}}^{\prime} \leq \min \left[\frac{\varepsilon_{j}}{2}, 2^{-j}\right], \quad\left|K_{N_{l}} \tilde{\psi}_{j+1}\right|_{m}^{\prime}<\eta, \quad l=1, \ldots, j,
$$

and

$$
\left|K_{N_{j+1}} \tilde{\psi}_{j+1}\right|_{m}^{\prime}>\left|K_{N_{j+1}} \psi_{N_{j+1}}\right|_{m}^{\prime}-\frac{\varepsilon}{3}>\frac{2 \varepsilon}{3} .
$$

Define $\phi_{j+1}:=\phi_{j}+\tilde{\psi}_{j+1}$. Then, for $n=1, \ldots, j$,

$$
\left|K_{N_{n}} \phi_{j+1}\right|_{m}^{\prime} \geq\left|K_{N_{n}} \phi_{j}\right|_{m}^{\prime}-\left|K_{N_{n}} \tilde{\psi}_{j+1}\right|_{m}^{\prime}>\left(\frac{\varepsilon}{3}+\eta\right)-\eta=\frac{\varepsilon}{3},
$$

while, since $\left|K_{N_{j+1}} \phi_{j}\right|_{m}^{\prime} \leq \varepsilon / 3$,

$$
\left|K_{N_{j+1}} \phi_{j+1}\right|_{m}^{\prime} \geq\left|K_{N_{j+1}} \tilde{\psi}_{j+1}\right|_{m}^{\prime}-\left|K_{N_{j+1}} \phi_{j}\right|_{m}^{\prime}>\frac{2 \varepsilon}{3}-\frac{\varepsilon}{3}=\frac{\varepsilon}{3} .
$$

Thus (A.2) holds with $j$ replaced by $j+1$. Further,

$$
\left|\phi_{j+1}-\phi_{j}\right|_{n_{j}}^{\prime}=\left|\tilde{\psi}_{j+1}\right|_{n_{j}}^{\prime} \leq 2^{-j} .
$$

Finally, for all $\chi \in Y$ with $|\chi|_{n_{j+1}}^{\prime} \leq \varepsilon_{j+1}$, it follows from (A.4) that $\left\|\phi_{j+1}+\chi\right\|_{\infty}=\left\|\phi_{j}+\left(\tilde{\psi}_{j+1}+\chi\right)\right\|_{\infty} \leq \max \left(M,\left\|\tilde{\psi}_{j+1}+\chi\right\|_{\infty}\right)+2-2^{1-j}$, since

$$
\left|\tilde{\psi}_{j+1}+\chi\right|_{n_{j}}^{\prime} \leq\left|\tilde{\psi}_{j+1}\right|_{n_{j}}^{\prime}+|\chi|_{n_{j+1}}^{\prime} \leq \frac{\varepsilon_{j}}{2}+\varepsilon_{j+1} \leq \varepsilon_{j} .
$$


Further,

$$
\left\|\tilde{\psi}_{j+1}+\chi\right\|_{\infty} \leq \max \left(M,\|\chi\|_{\infty}\right)+2^{-j}
$$

Thus

$$
\begin{aligned}
\left\|\phi_{j+1}+\chi\right\|_{\infty} & \leq \max \left(M,\|\chi\|_{\infty}\right)+2+2^{-j}-2^{1-j} \\
& =\max \left(M,\|\chi\|_{\infty}\right)+2-2^{1-(j+1)},
\end{aligned}
$$

i.e., (A.4) holds with $j$ replaced with $j+1$.

We have shown above that it is possible to construct sequences $\left(\phi_{j}\right) \subset Y,\left(\varepsilon_{j}\right) \subset(0, \infty)$ and strictly monotonic sequences $\left(N_{j}\right) \subset \mathbf{N}$ and $\left(n_{j}\right) \subset \mathbf{N}$, satisfying (A.2), (A.3) and (A.4). Note that (A.4) implies that $\left\|\phi_{j}\right\|_{\infty} \leq M+2, j \in \mathbf{N}$, so that $\left(\phi_{j}\right)$ is norm (and $\beta-$ ) bounded. Further, (A.3) implies that, if $j, k, l \in \mathbf{N}, l>j$, and $n_{j} \geq k$,

$$
\left|\phi_{l}-\phi_{j}\right|_{k}^{\prime} \leq \frac{1}{2^{j}}+\cdots+\frac{1}{2^{l-1}}<\frac{1}{2^{j-1}} .
$$

Thus $\left(\phi_{j}\right)$ is $\tau$-Cauchy. Hence, since $X$ is a Fréchet space and applying Theorem 2.1 (ii), $\left(\phi_{j}\right)$ is $\tau$-convergent to some $\phi \in Y$. Since $\left(\phi_{j}\right)$ is also $\beta$-bounded, $\phi_{j} \stackrel{\beta}{\rightarrow} \phi$, by Theorem 2.1 (iv). Now, using (A.2),

$$
\left|K_{N_{n}} \phi\right|_{m}^{\prime}>\frac{\varepsilon}{3}-\left|K_{N_{n}}\left(\phi-\phi_{j}\right)\right|_{m}^{\prime}
$$

for $j=n, n+1, \ldots$, and since $K_{N_{n}} \in S(Y)$ and $\phi_{j} \stackrel{\beta}{\rightarrow} \phi, \mid K_{N_{n}}(\phi-$ $\left.\phi_{j}\right)\left.\right|_{m} ^{\prime} \rightarrow 0$ as $j \rightarrow \infty$. Thus

$$
\left|K_{N_{n}} \phi\right|_{m}^{\prime} \geq \frac{\varepsilon}{3}, \quad n \in \mathbf{N}
$$

This contradicts $K_{n} \stackrel{S}{\rightarrow} 0$ and the proof is completed.

Lemma A.4. Suppose $K_{n} \in S(Y), n \in \mathbf{N}$. Then $\mathcal{K}:=\left\{K_{n}: n \in\right.$ $\mathbf{N}\}$ is $\beta$-sequentially equicontinuous if and only if

$$
\left(\psi_{n}\right) \subset Y, \psi_{n} \stackrel{\beta}{\rightarrow} 0 \Longrightarrow K_{n} \psi_{n} \stackrel{\beta}{\rightarrow} 0 .
$$

Proof. Clearly, if $\mathcal{K}$ is $\beta$-sequentially equicontinuous, then (A.6) holds. To show the converse, suppose the sequence $\left(L_{n}\right) \subset \mathcal{K}$ and 
that $\left(\psi_{n}\right) \subset Y, \psi_{n} \stackrel{\beta}{\rightarrow} 0$. Then the proof will be completed if we can show that $L_{n} \psi_{n} \stackrel{\beta}{\rightarrow} 0$. It is enough to show that every subsequence of $\left(L_{n} \psi_{n}\right)$ has a subsequence which is $\beta$-convergent to zero. So consider a subsequence of $\left(L_{n} \psi_{n}\right)$, denoted again by $\left(L_{n} \psi_{n}\right)$. This has a subsequence $\left(L_{n_{m}} \psi_{n_{m}}\right)$ such that either $L_{n_{m}}=K_{j}$, for some $j \in N$ and all sufficiently larger $m$, or $L_{n_{m}}=K_{j_{m}}$, with $j_{m} \rightarrow \infty$ as $m \rightarrow \infty$. In the first case $L_{n_{m}} \psi_{n_{m}} \rightarrow 0$ since $K_{j} \in S(Y)$. In the second case this follows from (A.6).

Proof of Theorem 3.8. Suppose that Assumption A holds and that $K \in S(Y), K_{n} \in S(Y), n \in \mathbf{N}$ and $K_{n} \stackrel{S}{\rightarrow} K$. Define $\tilde{K}_{n} \in S(Y)$ by $\tilde{K}_{n}:=K_{n}-K$. Then $\tilde{K}_{n} \stackrel{S}{\rightarrow} 0$ and, by Theorem A.3, $\tilde{K}_{n} \stackrel{\beta}{\rightarrow} 0$. It follows from Lemma A.4 that $\left\{\tilde{K}_{n}: n \in \mathbf{N}\right\}$ is $\beta$-sequentially equicontinuous. Since $K \in S(Y),\left\{K_{n}: n \in \mathbf{N}\right\}$ is $\beta$-sequentially equicontinuous.

In this last lemma and the proof of Theorem 3.7 we use the notations $M_{r}:=\left\{\phi \in Y:\|\phi\|_{\infty}<r\right\}$, for $r>0$, and $B_{\varepsilon}^{a}:=\left\{\phi \in Y:|\phi|_{a}<\varepsilon\right\}$, for $\varepsilon>0, a \in S_{0}$.

Lemma A.5. Suppose Assumption $\mathrm{A}^{\prime}$ holds, $L \in S(Y), U \subset Y$ is $\tau$-open and convex with $0 \in U$ and $L\left(M_{r} \cap U\right) \subset B_{\varepsilon}^{a}$ for some $a \in S_{0}$. Then, for every $\eta>\varepsilon$, there exists $N \in \mathbf{N}$ such that

$$
L\left(M_{r+1} \cap\left\{\phi \in Y:|\phi|_{N}^{\prime}<r\right\} \cap U\right) \subset B_{\eta}^{a} .
$$

Proof. First note that

$$
L\left(M_{2 r+1} \cap Y_{l}\right) \subset B_{\eta-\varepsilon}^{a}
$$

for some $l \in \mathbf{N}$. For if this does not hold then for every $l \in \mathbf{N}$ there exists $\phi_{l} \in M_{2 r+1} \cap Y_{l}$ with $L \phi_{l} \notin B_{\eta-\varepsilon}^{a}$. But then $\phi_{l} \stackrel{\beta}{\rightarrow} 0$ as $l \rightarrow \infty$ which implies $L \phi_{l} \stackrel{\beta}{\rightarrow} 0$, contradicting that $L \phi_{l} \notin B_{\eta-\varepsilon}^{a}, l \in \mathbf{N}$.

Choose $l$ so that (A.7) holds and so that $Y_{l} \subset U$ (note that $Y_{l} \subset U$ for all sufficiently large $l$ since $U$ is $\tau$-open and $0 \in U$ ). Since Assumption $\mathrm{A}^{\prime}$ holds, there exist $N>l$ and $P: Y \rightarrow Y_{l}$ such that

$$
\|\phi-P \phi\|_{\infty} \leq|\phi|_{N}^{\prime}, \quad \phi \in Y \text {. }
$$


Let $U_{N}:=\left\{\phi \in Y:|\phi|_{N}^{\prime}<r\right\} \cap U$. Then, for all $\psi \in M_{r+1} \cap U_{N}$,

$$
\|P \psi\|_{\infty} \leq\|P \psi-\psi\|_{\infty}+\|\psi\|_{\infty} \leq|\psi|_{N}^{\prime}+r+1<2 r+1 .
$$

Thus $P \psi \in M_{2 r+1} \cap Y_{l}$. Since $\psi \in U_{N}$ it follows from (A.8) that $\psi-P \psi \in M_{r}$. Further, $t \psi \in U$ for some $t>1$ since $U$ is open, and $-s P \psi \in Y_{l} \subset U$ for every $s>0$, so that $\psi-P \psi=\frac{1}{t}(t \psi)+$ $\frac{t-1}{t}\left(-\frac{t}{t-1} P \psi\right) \in U$, as $U$ is convex. Thus

$$
\begin{aligned}
L \psi & =L(P \psi)+L(\psi-P \psi) \in L\left(M_{2 r+1} \cap Y_{l}\right)+L\left(M_{r} \cap U\right) \\
& \subset B_{\eta-\varepsilon}^{a}+B_{\varepsilon}^{a} \subset B_{\eta}^{a} .
\end{aligned}
$$

Proof of Theorem 3.7. We have to show that if Assumption $\mathrm{A}^{\prime}$ holds and $L$ is $\beta$-sequentially continuous, then, for every $a \in S_{0}$ and $\varepsilon>0$ there exists a $\beta$-open set $U$ with $L(U) \subset B_{\varepsilon}^{a}$. We show that such a set $U$ exists by a recursive construction.

For $n \in \mathbf{N}$, let $p_{n}:=1-(2 n)^{-1}$. As a first step choose $\delta>0$ such that $L\left(M_{\delta}\right)=\delta L\left(M_{1}\right) \subset B_{p_{1} \varepsilon}^{a}$. This is possible since $L \in$ $S(Y) \subset B(Y)$ so that $L\left(M_{1}\right)$ is bounded and so absorbed by $B_{p_{1} \varepsilon}^{a}$. Set $U_{1}:=\left\{\phi:|\phi|_{1}^{\prime}<\delta\right\}$. Then $U_{1}$ is $\tau$-open and convex, $0 \in U_{1}$ and $L\left(M_{\delta} \cap U_{1}\right)=L\left(M_{\delta}\right) \subset B_{p_{1} \varepsilon}^{a}$.

For $n \in \mathbf{N}$ suppose $U_{n}$ has been constructed which is $\tau$-open and convex and satisfies $0 \in U_{n}$ and $L\left(M_{\delta+n-1} \cap U_{n}\right) \subset B_{p_{n} \varepsilon}^{a}$. Choose $N_{n} \in \mathbf{N}$ such that

$$
L\left(M_{\delta+n} \cap\left\{\phi:|\phi|_{N_{n}}^{\prime}<\delta+n-1\right\} \cap \cup_{n}\right) \subset B_{p_{n+1} \varepsilon}^{a}
$$

(which is possible by Lemma A.5) and set $U_{n+1}:=\left\{\phi:|\phi|_{N_{n}}^{\prime}<\right.$ $\delta+n-1\} \cap U_{n}$. Then $U_{n+1}$ is $\tau$-open and convex, $0 \in U_{n+1}$ and $L\left(M_{\delta+n} \cap U_{n+1}\right) \subset B_{p_{n+1} \varepsilon}^{a}$.

The sequence of sets $U_{1}, U_{2}, \ldots$ obtained by this construction satisfies $M_{\delta+n-1} \cap U_{n} \subset M_{\delta+n} \cap U_{n+1}$ and $L\left(M_{\delta+n-1} \cap U_{n}\right) \subset B_{p_{n} \varepsilon}^{a}$, for $n \in \mathbf{N}$, so that $L(U) \subset B_{\varepsilon}^{a}$, where

$$
U:=\bigcup_{n=1}^{\infty}\left(M_{\delta+n-1} \cap U_{n}\right) .
$$


Further,

$$
U=\bigcap_{n=1}^{\infty} U_{n}=\left\{\phi:|\phi|_{N_{1}}^{\prime}<\delta,|\phi|_{N_{2}}^{\prime}<\delta+1, \ldots\right\},
$$

so that $U=B_{1}^{\tilde{a}}$, for some $\tilde{a} \in S_{0}$. Thus $U$ is $\beta$-open.

\section{REFERENCES}

1. R.P. Agarwal and D.O'Regan, Infinite interval problems for differential, difference and integral equations, Kluwer, Dordrecht, 2001.

2. P.M. Anselone, Collectively compact operator approximation theory and applications to integral equations, Prentice-Hall, Englewood Cliffs, 1971.

3. P.M. Anselone and J.W. Lee, Nonlinear integral equations on the half-line, J. Integral Equations Appl. 4 (1992), 1-14.

4. P.M. Anselone and I.H. Sloan, Integral equations on the half-line, J. Integral Equations 9 (1985), 3-23.

5. T. Arens, The scattering of elastic waves by rough surface, Ph.D. thesis, Brunel University, 2000.

6. K.E. Atkinson, The numerical solution of integral equations on the half-line, SIAM J. Numer. Anal. 6 (1969), 375-397.

7. R.C. Buck, Bounded continuous functions on a locally compact space, Michigan Math. J. 5 (1958), 95-104.

8. S.N. Chandler-Wilde, Some uniform stability and convergence results for integral equations on the real line and projection methods for their solution, IMA J. Numer. Anal. 13 (1993), 509-535.

9. S.N. Chandler-Wilde and A.T. Peplow, Asymptotic behavior at infinity of solutions of multidimensional second kind integral equations, J. Integral Equations Appl. 7 (1995), 303-327.

10. S.N. Chandler-Wilde and B. Zhang, On the solvability of a class of second kind integral equations on unbounded domains, J. Math. Anal. Appl. 214 (1997), 482-502.

11. - Scattering of electromagnetic waves by an inhomogeneous conducting or dielectric layer, SIAM J. Math. Anal. 30 (1999), 559-583.

12. S.N. Chandler-Wilde, B. Zhang and C.R. Ross, On the solvability of second kind integral equations on the real line, J. Math. Anal. Appl. 245 (2000), 28-51.

13. D. O'Regan, Approximation of solutions of nonlinear operator equation on the half-line, Computers Math. Appl. 35 (1998), 65-77.

14. A.T. Peplow and S.N. Chandler-Wilde, Approximate solution of second kind integral equations on infinite cylindrical surfaces, SIAM J. Numer. Anal. 32 (1995), 594-609.

15. A.P. Robertson and W.J. Robertson, Topological vector spaces, 2nd ed., Cambridge University Press, Cambridge, 1973. 
16. W. Rudin, Functional analysis, 2nd ed., McGraw-Hill, New York, 1991.

17. - Real and complex analysis, 3rd ed., McGraw-Hill, New York, 1987.

18. B. Zhang and S.N. Chandler-Wilde, Acoustic scattering by an inhomogeneous layer on a rigid plate, SIAM J. Appl. Math. 58 (1998), 1931-1950.

19. R.J. Zimmer, Essential results of functional analysis, The University of Chicago Press, Chicago, 1990.

Department of Mathematical Sciences, Brunel University, Uxbridge UB8 3PH, UK

E-mail address: Simon.Chandler-Wilde@brunel.ac.uk

School of Mathematical and Information Sciences, Coventry UniverSity, Coventry CV1 5FB, UK

E-mail address: B.Zhang@coventry.ac.uk 